LBNL-59069

UCB-PTH-05/38

UPR-1136-T

\title{
On Quadratic Divergences in Supergravity, Vacuum Energy and the Supersymmetric Flavor Problem
}

\author{
Mary K. Gaillard ${ }^{1}$ and Brent D. Nelson ${ }^{2}$ \\ ${ }^{1}$ Department of Physics, University of California \\ and \\ Theoretical Physics Group, Lawrence Berkeley National Laboratory, Berkeley, CA 94720, USA \\ ${ }^{2}$ Department of Physics and Astronomy, \\ University of Pennsylvania, Philadelphia, PA 19104, USA
}

Dated: November 18, 2005

\begin{abstract}
We examine the phenomenological consequences of quadratically divergent contributions to the scalar potential in supergravity effective Lagrangians. We focus specifically on the effect of these corrections on the vacuum configuration of scalar fields in softly-broken supersymmetric theories and the role these corrections play in generating non-diagonal soft scalar masses. Both effects can only be properly studied when the divergences are regulated in a manifestly supersymmetric manner - something which has thus far been neglected in past treatments. We show how a supersymmetric regularization can impact past conclusions about both types of phenomena and discuss what types of high-energy theories are likely to be safe from unwanted flavor-changing neutral current interactions in the context of supergravity theories derived from heterotic string compactifications.
\end{abstract}




\section{Disclaimer}

This document was prepared as an account of work sponsored by the United States Government. Neither the United States Government nor any agency thereof, nor The Regents of the University of California, nor any of their employees, makes any warranty, express or implied, or assumes any legal liability or responsibility for the accuracy, completeness, or usefulness of any information, apparatus, product, or process disclosed, or represents that its use would not infringe privately owned rights. Reference herein to any specific commercial products process, or service by its trade name, trademark, manufacturer, or otherwise, does not necessarily constitute or imply its endorsement, recommendation, or favoring by the United States Government or any agency thereof, or The Regents of the University of California. The views and opinions of authors expressed herein do not necessarily state or reflect those of the United States Government or any agency thereof of The Regents of the University of California and shall not be used for advertising or product endorsement purposes.

Lawrence Berkeley Laboratory is an equal opportunity employer. 


\section{Introduction}

The supersymmetric flavor problem [1, 2] continues to lie at the heart of phenomenological treatments of supersymmetry breaking. The apparently small size of off-diagonal mass terms in the effective potential in any softly broken supersymmetric model has often been cited as particularly troublesome for models in which supergravity plays a prominent role in transmission of supersymmetry breaking to the observable sector - and therefore for string-inspired models more generally [3].

It is important, however, to distinguish "general gravity mediation" from the specific manifestations that arise in string models. In a completely general model of gravity mediation there is indeed a generic "flavor problem," irrespective of the quadratic divergence issue. That is to say that one can imagine a priori operators of the form

$$
\int \mathrm{d}^{4} \theta \frac{\bar{X} X}{M_{\mathrm{PL}}^{2}} \bar{Q}^{\bar{i}} Q^{j}
$$

where $X$ is a Standard Model singlet that is presumably a hidden sector field. If it participates in supersymmetry (SUSY) breaking, then it will generate off-diagonal soft-masses. The key is that without specifying a rule for how this $X$ couples to Standard Model matter (such as via gauge charges in gauge mediation) then we must assume that different flavors can be treated differently. Rephrased in a manner common to treatments of the flavor problem in supersymmetry: there is no symmetry argument as to why operators of the form of (1) should be absent.

But the symmetry here being considered is typically that of a gauge symmetry. Yet the operator in (11) may admit a geometrical interpretation. Let us rewrite things to make this more apparent

$$
\int \mathrm{d}^{4} \theta \frac{R_{\bar{i} j \bar{k} \ell}}{M_{\mathrm{PL}}^{2}} \bar{X}^{\bar{k}} X^{\ell} \bar{Q}^{\bar{i}} Q^{j},
$$

where the tensor $R_{\bar{i} j \bar{k} \ell}$ is the curvature tensor formed from the field-reparameterization connection

$$
R_{j k \bar{m}}^{i}=D_{\bar{m}} \Gamma_{j k}^{i} ; \quad \Gamma_{j k}^{i}=K^{i \bar{n}} \partial_{j} K_{k \bar{n}}=K^{i \bar{n}} \partial_{j} \partial_{k} \partial_{\bar{n}} K
$$

where $D_{\bar{m}}=K_{\ell \bar{m}} D^{\ell}$ is a covariant derivative with respect to field reparameterization and $K_{\ell \bar{m}}$ is the Kähler metric. Thus, while an understanding of the form of this tensor in (2) may not be possible in terms of the gauge quantum numbers of the fields involved, an understanding in terms of the isometries of the manifold defined by the chiral superfields of the theory may indeed exist. This point has been emphasized recently for string-based effective supergravity theories [4, 5].

In the absence of an underlying theory we typically proceed by making assumptions. For example, we can achieve the minimal supergravity paradigm by assuming the curvature tensor 
factorizes as $R_{\bar{i} j \bar{k} \ell} \propto K_{\bar{i} j} K_{\bar{k} \ell}$ and $K_{\bar{i} j} \propto \delta_{\bar{i} j}$ [6] [7. In string-based models there may be a basis in which the Kähler metric for the matter fields is diagonal in its generation indices [8]. This need not be the same basis in which fermion masses are diagonal, but this is the standard problem that all methods of SUSY breaking share. Then the question is whether higher order terms such as (2) are the result of terms at higher genus in the string loop expansion (and thus require a string-theory explanation) or are the result of field-theory loop corrections involving gravitationalstrength interactions. In the case of the latter, the all-important matrix $R_{\bar{i} j \bar{k} \ell}$ can be determined from the tree-level theory.

But the supergravity flavor problem is often considered even more severe than the discussion above would indicate. As a non-renormalizable effective theory, supergravity models suffer corrections to their effective potentials that grow quadratically with some high mass scale. The structure of these loop-induced contributions to the effective potential is determined by the structure of the tree-level Kähler potential for matter fields. While these corrections come with a loop-factor relative to the leading-order contributions, it has been noted that they are often also proportional to the number of light fields in the theory [9, 10, which can be quite large for string-based models. Thus, it is argued, it may not be sufficient to ensure the absence of dangerous terms such as (2) at the leading order. As these terms also contribute to the vacuum expectation value of the effective potential, their presence has been conjectured to play a significant role in determining the vacuum configuration of light fields in the theory.

In this paper we re-visit these issues, taking care to address them only after a proper (supersymmetric) regularization of the apparent divergences is performed. This important step has been completely overlooked in previous treatments of the phenomenological implications of quadratic divergences. In Section 1 below we exhibit the divergent contributions to the effective potential in a non-technical manner and discuss the importance of a proper regularization scheme. We intend for the treatment in that section to be accessible to the non-expert. In Section 2 we present the technical details, first of the quadratic divergences themselves in Section 2.1 and then in Section 2.2 of the Pauli-Villars (PV) regulator sector we will use to cancel the divergences and render these contributions finite. In Section 2.3 we demonstrate explicitly how the supersymmetric regularization scheme allows for an interpretation of the loop corrections as a supersymmetric renormalization of the space-time metric and Kähler potential themselves. The reader uninterested in this level of technical detail can proceed directly to Section 3, where the phenomenological implications of these loop corrections for vacuum stabilization and scalar masses are treated. This section is largely self-contained, with only the occasional need to refer to certain key results from Section 2. 


\section{Divergent Contributions at One Loop to the Effective Scalar Potential}

The effective potential at one loop for a generic theory is given by [11] - [16]

$$
V_{1-\text { loop }}=V_{0}+\frac{1}{64 \pi^{2}} \mathrm{~S} \operatorname{Tr} \mathcal{M}^{0} \cdot \Lambda^{4} \ln \frac{\Lambda^{2}}{\mu^{2}}+\frac{1}{32 \pi^{2}} \mathrm{~S} \operatorname{Tr} \mathcal{M}^{2} \cdot \Lambda^{2}+\frac{1}{64 \pi^{2}} \mathrm{~S} \operatorname{Tr} \mathcal{M}^{4} \cdot \ln \frac{\mathcal{M}^{2}}{\Lambda^{2}}+\text { finite }
$$

where we define the quantity

$$
\mathrm{S} \operatorname{Tr} \mathcal{M}^{n} \equiv \sum_{i}(-1)^{2 J_{i}} C\left(J_{i}\right) m_{i}^{n}
$$

with $m_{i}$ being the (possibly field-dependent) mass of the state in the summation, and $C\left(J_{i}\right)=2 J_{i}+1$ for $J_{i} \leq 1$. The definition of the supertrace over the degrees of freedom in the gravity sector depends on the gauge-fixing procedure utilized [17. ${ }^{1}$ The first term $V_{0}$ is the tree-level (classical) scalar potential. The second quantity is, strictly speaking, the loop-induced "vacuum energy" in the limited sense that it is a contribution to the scalar potential that scales like the fourth power of the cut-off $\Lambda$ with a field-independent coefficient. In any model where the spectrum obeys $N=1$ supersymmetry this coefficient vanishes identically. The third and fourth terms in (41) are the quadratic and logarithmic divergences, respectively. These were computed for a general supergravity Lagrangian in [12] - 19] and for specific heterotic string models with modular invariance in [14, 20, 21].

Before proceeding to the issue of scalar mass and flavor-changing effects in the low-energy theory, let us pause for a brief digression on the issue of vacuum energy in these theories. Given that we expect the masses of heavy fields in the theory to be on the order of the gravitino mass such that $m_{i} \sim m_{3 / 2}$, we might imagine that the quadratically divergent term in (4) is providing a contribution to the vacuum energy in an amount $\left\langle\delta V_{1-\text { loop }}\right\rangle \simeq m_{3 / 2}^{2} M_{\mathrm{PL}}^{2}$. For consistency with cosmological observations (and for the sake of doing a meaningful phenomenological study of effective Lagrangians) we are often at pains to engineer the vanishing of the vacuum expectation value (vev) of the tree-level scalar potential: $\left\langle V_{0}\right\rangle=0$. A straightforward interpretation of (4) would seem to suggest that the one-loop contribution to this effective vacuum energy is generally positive and that we should therefore strive to engineer $\left\langle V_{0}\right\rangle<0$. This was the standpoint of [9], where it was suggested that this contribution to the vacuum energy might compensate for the large negative contribution that typically arises in the so-called "racetrack" models of moduli stabilization.

\footnotetext{
${ }^{1}$ For example $C\left(\frac{3}{2}\right)=+4$ in the gauge of [17 18, 19] that is used in the results quoted below.
} 
It is certainly true that whatever mechanism Nature employs in ensuring a vanishing (or nearly vanishing) cosmological constant must do so to amazing accuracy; surely a phenomenological model of this mechanism (such as the minimization of some potential for a modulus which couples to these various contributions) must take into account these loop-induced terms. The presence of the loop factors in (4) might suggest that these higher-order contributions are small perturbations on the result that causes the classical vacuum energy to vanish. But, as was pointed out in [9], the sum over multiplets implied in the supertrace brings a potentially large number into the one-loop contribution. For example, in a simple model with only $N_{\chi}$ chiral superfields, $N_{G}$ Yang-Mills multiplets, and gravity we would have [17, 19]

$$
\left\langle\delta V_{1-\mathrm{loop}}\right\rangle \sim \frac{\Lambda^{2}}{32 \pi^{2}} \mathrm{~S} \operatorname{Tr} \mathcal{M}^{2} \simeq \frac{\Lambda^{2}}{16 \pi^{2}}\left(N_{\chi} m_{0}^{2}-N_{G} m_{1 / 2}^{2}+2 m_{3 / 2}^{2}\right)
$$

with the coefficient of the last term in (6) depending on the gauge-fixing prescription employed. For the MSSM field content (without right-handed neutrinos) these coefficients are $N_{\chi}=49$ and $N_{G}=12$. For the much larger field content of a typical $Z_{3}$ orbifold compactification of the $E_{8} \times E_{8}$ heterotic string we might have 300 or more chiral multiplets while the gauge multiplets are typically bounded with $N_{G} \lesssim 65[22$.

But the conclusion that this contribution is then typically positive is a naive one. In particular, the form of (6) implies a common cut-off for each term, whereas these cut-offs will vary from term to term and may be field-dependent. Indeed, only by employing field-dependent cut-offs can supersymmetry be maintained in the presence of loop corrections to the effective potential [23] [26. When the effective cut-off is field-dependent it is no longer correct to assume that $\Lambda_{\text {eff }}^{2}>0$ in all cases. In fact, even if the effective cut-offs are constants, four-dimensional supergravity theories require a priori at least two subtractions to render the divergent loop integrals finite. In the context of Pauli-Villars regularization this is equivalent to requiring at least two sets of fields for every one light field. Proper regularization of the theory - which is to say, elimination of divergences - thus results in the replacement

$$
\Lambda^{2} \mathrm{~S} \operatorname{Tr} \mathcal{M}^{2} \rightarrow \mathrm{S} \operatorname{Tr} \mu^{2} \mathcal{M}^{2} \ln \left(\mu^{2}\right) \eta_{S}, \quad \eta_{S}=\sum_{q} \eta_{q} \lambda_{q} \ln \lambda_{q}
$$

The quantity $\eta_{S}$ is a coefficient that represents the uncertainty in the threshold for the onset of this new physics and $\eta_{q}= \pm 1$ is chosen to ensure finiteness of the effective Lagrangian. Note that we can define the quantity $\mu_{q}^{2}=\lambda_{q} \mu^{2}, \lambda_{q}>0$ which represents the mass-scale of the new physics corresponding to each of the $q$ subtractions (or PV superfields $\Phi^{q}$ ) that are serving as regulators to 
the supergravity theory. For the case of regularization via Pauli-Villars (PV) regulators, which we will employ in the next section, these threshold factors can be computed in terms of the properties of the PV sector. The sign of the effective cut-off is determined by the sign of the factor $\eta_{S}$.

It turns out that the cancellation of all ultraviolet (UV) divergences in realistic string-derived supergravity models requires at least 5 PV supermultiplets for each supermultiplet of the low energy theory [27. The important point is that with four or more subtractions it is not necessary for the coefficient $\eta_{S}$ to be positive. ${ }^{2}$ As this is an important point, let us take a moment to sketch its derivation. The cancellation of quadratic divergences imposes the following conditions [28] on the sum over signatures

$$
\sum_{q=1}^{S} \eta_{q}=-1, \quad \sum_{q} \eta_{q} \lambda_{q}=0
$$

where $S \geq 2$ is the total number of subtractions (PV superfields). The first equality requires $S$ odd: $S=2 n+1 \geq 3$. In the simplest case where $S=3, \eta_{q}=(-1,-1,1)$, and $\lambda_{q}=\left(\lambda_{1}, \lambda_{2}, \lambda_{1}+\lambda_{2}\right)$, we have

$$
\eta_{S}=-\lambda_{1} \ln \lambda_{1}-\lambda_{2} \ln \lambda_{2}+\left(\lambda_{1}+\lambda_{2}\right) \ln \left(\lambda_{1}+\lambda_{2}\right)>0 .
$$

However for $n \geq 1, S \geq 5, \eta_{S}$ is not positive definite. To see this take the following example

$$
\begin{aligned}
\eta_{1}, \cdots \eta_{n}=1, & \eta_{n+1}, \cdots \eta_{2 n+1}=-1, \\
\lambda_{1} \cdots \lambda_{n}=\Lambda / n, & \lambda_{n+1} \cdots \lambda_{2 n}=\lambda, \quad \lambda_{2 n+1}=\Lambda-n \lambda .
\end{aligned}
$$

Then

$$
\eta_{S}=\Lambda \ln (\Lambda / n)-n \lambda \ln \lambda-(\Lambda-n \lambda) \ln (\Lambda-n \lambda)=\lambda f(\Lambda / \lambda, n)
$$

Now since

$$
\lim _{x \rightarrow \infty} f(x, n)=-x \ln n+n \ln x+O\left(x^{-1}\right)
$$

is negative for $n>1$, the function $\eta_{S}$ is positive definite only for $n=1, S=3$. Cancellation of all the ultraviolet divergences of a general supergravity theory requires at least $5 \mathrm{PV}$ chiral multiplets for every light chiral multiplet and even more PV supermultiplets to regulate the gauge loops. Therefore one cannot assume that the effective cut-offs are positive.

In general, using a straight cut-off regulator is equivalent to an explicit breaking of supersymmetry and is thus inconsistent with the requirement that supersymmetry be broken only spontaneously by the vevs of fields in the low-energy theory. Rather, these cut-offs should be regarded as fielddependent. Indeed, it is the very field-dependence of these cut-offs that allows for the possibility of

\footnotetext{
${ }^{2}$ See, for example, the discussion of this point in Appendix C of [28].
} 
a dynamical mechanism for cancelling the vacuum energy to arbitrarily high loop-order. We will discuss an example of this possibility, including the effect of the one-loop correction in Section 3.1 below. Here we wish to briefly describe the nature of this field dependence. Supersymmetric PauliVillars regularization of matter and gauge loops ${ }^{3}$ is implemented by introducing supersymmetric masses for PV fields $\Phi^{A}, \Pi^{A}$ through superpotential couplings of the form

$$
W_{\mathrm{PV}}=\mu_{A B}\left(Z^{i}\right) \Phi^{A} \Pi^{B}
$$

where $\mu_{A B}$ is a holomorphic function of the light chiral multiplets $Z^{i}$. Then the effective squared cut-off is replaced by the (signature-weighted) squared PV mass matrix:

$$
\Lambda_{\text {eff }}^{2} \rightarrow\left(M_{\mathrm{PV}}^{2}\right)_{B}^{A}=e^{K} K_{\Phi}^{A \bar{C}} K_{\Pi}^{D \bar{E}} \bar{\mu}_{\bar{C} \bar{E}}(\bar{Z}) \mu_{B D}(Z) .
$$

By convention we denote by $\Phi^{A}$ those fields that regulate divergences arising from the gauge and superpotential couplings of the light fields; their Kähler metric $K_{A \bar{B}}^{\Phi}$ is determined by the effective supergravity theory of the light fields. ${ }^{4}$ The Kähler metric for the fields $\Pi$, introduced to generate supersymmetric PV masses, is much less constrained. For example if we took

$$
\mu_{A B}=\mu \delta_{A B}, \quad K_{A \bar{B}}^{\Pi}=e^{-K}\left(K_{\Phi}^{-1}\right)_{A \bar{B}},
$$

with $\mu$ constant, the effective cut-off would indeed be constant. This may seem like a very convenient choice from the point of view of the effective field theory. Considerations of the anomaly structure of the effective theory in string-based models may make such constant effective cut-offs inconsistent. In the effective field theory of heterotic orbifold models [29, 30, for example, the anomaly structure does not allow for constant cut-offs [25]. Since superpotential couplings depend on moduli in a general string model, this is likely to be a generic feature of effective supergravity models which seek to describe their low-energy behavior. As we will see in Section 3. this dependence is crucially important for the low-energy phenomenology of supergravity effective theories at the one-loop level. We will have more to say about the implications of (14) in the conclusion section of this work.

But there is a deeper reason why the cut-offs in (4) should be regarded as field-dependent and not straight cut-offs. In addition to the substitution $\Lambda^{2} \rightarrow M_{\mathrm{PV}}^{2}$ in the cut off theory, the presence of the superpotential (13) induces additional terms in the regulated theory such that the net result of the quadratically divergent contributions is a renormalization of the space-time metric and the Kähler

\footnotetext{
${ }^{3}$ As noted below full regularization of gravity loops requires the introduction of massive PV (Abelian) vector multiplets as well.

${ }^{4}$ We will use upper-case letters to collectively denote the fields in the PV sector throughout this work.
} 
potential itself. By simply inserting a cut-off in the divergent integrals one would not generate these additional terms that are needed for supersymmetry [28, 26]. Put differently, the use of straight cutoffs breaks the local supersymmetry of the supergravity Lagrangian and prevents us from treating the renormalization at the superspace level. As will be made explicit in Section 2.3 below, the interpretation of the one-loop quadratic divergences in terms of renormalizations is valid only to lowest order in the loop expansion parameter $\epsilon=\hbar / 16 \pi^{2}$. If these terms have very large coefficients, all the leading $\left(\epsilon \Lambda_{\text {eff }}^{2}\right)^{n}$ terms must be retained so as to maintain manifest supersymmetry; the full correct result is easily inferred from the one-loop calculation.

Finally, let us note that nowhere do we make the artificial distinction between contributions to vacuum energy arising from "hidden" versus "observable" sector fields. Such a separation of contributions serves no physical purpose and creates confusion: any dynamical mechanism engineered in the low-energy theory to produce vanishing vacuum energy at the tree or $n$-loop level should take into account contributions to (4) arising from all sectors of the theory, including effects from symmetry breaking at energy scales lower than that of supersymmetry breaking (i.e. electroweak symmetry breaking, the QCD phase transition, etc.), even though these will likely have negligible impact on the actual vacuum configuration of the fields involved. It is, after all, the entirety of vacuum energy that is constrained by the apparent flatness of the universe (or measured, if one likes, by the recession rate of supernovae).

Having treated the issue of vacuum energy let us return to our primary concern: corrections to the scalar potential that may produce dangerous flavor-changing operators. Within the supertrace $\mathrm{STr} \mathcal{M}^{2}$ of the quadratically divergent term in (4) we find terms such as

$$
\mathrm{S} \operatorname{Tr} \mathcal{M}^{2} \ni 2 e^{-K} R_{i \bar{j}} \bar{A}^{i} A^{\bar{j}}=2 R_{m \bar{n} i \bar{j}} K^{m \bar{n}} K^{i \bar{p}} K^{q \bar{j}} e^{K}\left(K_{\bar{p}} \bar{W}+\bar{W}_{\bar{p}}\right)\left(K_{q} W+W_{q}\right)
$$

where we have introduced the notation $A \equiv e^{K} W$ for later convenience. Several potentially dangerous contributions to the effective scalar potential are contained within (16), among them a term with the structure of (2)

$$
\mathcal{L}_{1-\text { loop }} \ni \frac{\Lambda^{2}}{16 \pi^{2}} K^{m \bar{n}} R_{m \bar{n} i \bar{j}}\left(e^{K}|W|^{2}\right) Q^{i} \bar{Q}^{\bar{j}}
$$

where the cut-off scale $\Lambda$ is understood to be measured in units of the reduced Planck mass, which we have set to unity. While this cut-off is presumably $\mathcal{O}(1)$ in these units, the loop factor is partially compensated by a potentially large number coming from the contraction on the first two indices in the curvature tensor in (17). This term in the one-loop effective Lagrangian has a form capable of producing an off-diagonal scalar soft mass term for the squarks, which may be of the 
same general size as the tree-level (and presumably diagonal) soft masses [10, 31]. In the most general supergravity theory we have no reason to expect that all elements of the curvature tensor are not populated. The question of whether or not such dangerous terms really exist in the low energy theory then becomes a question of the properties of the non-linear sigma model inherited from the underlying theory.

\section{Quadratic Divergences and Their Regulation}

Having established the crucial importance of the quadratically divergent contributions to (4) in a schematic way in the previous section, we now wish to exhibit the full structure of these terms in a properly regularized context. In order to do this we will need to introduce the Pauli-Villars sector in a more complete manner. This section provides the necessary technical details to understand the notation and origin of the results we present in Section 3, This is not a complete description of the PV technique in supergravity theories. We will make certain simplifications (which we point out) along the way that are made possible by our desire to study the quadratic divergences only a full treatment of the logarithmic as well as the quadratic divergences can be found in 18, 19.

Regularization of supergravity theories is no simple matter. The work that we attempt to summarize here spans over a decade of research. Even the regularization of a simple system - such as a non-linear sigma model without Yang-Mills fields in a curved background - requires several species of PV regulator fields. The proliferation of fields, both in number and type, grows rapidly when we come to consider the string-inspired models in which we are most interested (such as those with non-canonical gauge kinetic terms in which Yang-Mills fields couple to a dilaton field). This is an unfortunate yet unavoidable fact of working with supergravity effective theories. To guide the reader we first make a point about notation. In general, upper-case indices refer to fields in the PV sector, while lower-case indices refer to light fields of the theory. Fields such as the $\Phi^{P}$ introduced in (13) from the previous section are meant to represent all fields of a certain type: here all the chiral superfields $\Phi$ of the PV sector which regulate loops involving light fields $Z$. Within this set we might refer to the fields $\Phi^{I}$ as that subset that transform under the gauge group(s) in the same way as the field $Z^{i}$, or the fields $\Phi^{a}$ that transform as the adjoint under the particular group $\mathcal{G}_{a}$. To each of these subsets (or species) we occasionally must associate multiple copies, labeled by Greek indices $\alpha, \beta$, etc. These are the multiple copies that mimic the multiple insertions needed to regulate the theory, as mentioned in Section 1. We often suppress this index when it would be superfluous in a particular expression, but it should be understood to be present. 


\subsection{The Quadratically Divergent Contribution at One Loop}

Our starting point is the one-loop effective action, which can be determined from those terms quadratic in the quantum fields when the Lagrangian is expanded about an arbitrary background

$$
\mathcal{L}_{Q}=-\frac{1}{2} \Phi^{T} Z_{\Phi}\left(\hat{D}_{\Phi}^{2}+H_{\Phi}\right) \Phi+\frac{1}{2} \bar{\Theta} Z_{\Theta}\left(i \not D_{\Theta}-M_{\Theta}\right) \Theta+\mathcal{L}_{\mathrm{gh}}+\mathcal{L}_{\mathrm{Gh}}
$$

where the last two terms represent ghost and ghostino Lagrangians, respectively. The quantities $\Phi$ and $\Theta$ are column vectors which contain the quantum bosons and quantum fermions, respectively, of the theory. For example, $\Phi=\left(h_{\mu \nu}, \widehat{A}^{a}, \hat{Z}^{i}, \hat{\bar{Z}}^{\bar{m}}\right)$ contains the graviton, gauge and scalar quantum fields and is a $2 N_{\chi}+4 N_{G}+10$ component object. Similarly, with the gauge-fixing choice of [17] - 19], $\Theta$ is an $N_{\chi}+N_{G}+5$ Majorana fermion, while the ghost and ghostino contributions are equivalent to, respectively, -2 times the contribution of a $\left(4+N_{G}\right)$-component scalar and +2 times the contribution of a four-component scalar, such that supersymmetry of the off-shell spectrum is maintained. The matrix-valued covariant derivatives $D_{\Phi}$ and $D_{\Theta}$, metric factors $Z_{\Phi}$ and $Z_{\Theta}$, as well as the quantities $H_{\Phi}$ and $M_{\Theta}$ are defined in [17]. With the gauge-fixing prescription described in that work, the one-loop contribution to the effective action is

$$
\begin{aligned}
\mathcal{L}_{1} & =\frac{i}{2} \operatorname{Tr} \ln \left(\hat{D}_{\Phi}^{2}+H_{\Phi}\right)-\frac{i}{2} \operatorname{Tr} \ln \left(-i \not D_{\Theta}+M_{\Theta}\right)+i \operatorname{Tr} \ln \left(D_{\mathrm{gh}}^{2}+H_{\mathrm{gh}}\right)-i \operatorname{Tr} \ln \left(D_{\mathrm{Gh}}^{2}+H_{\mathrm{Gh}}\right) \\
& \equiv \frac{i}{2} \mathrm{~S} \operatorname{Tr} \ln \left(\hat{D}^{2}+H\right)+T_{-} .
\end{aligned}
$$

To obtain the second line of (19) we have split the fermionic contribution into a helicity-even piece given by

$$
\hat{D}_{\Theta}^{2}+H_{\Theta} \equiv\left(-i \not D_{\Theta}+M_{\Theta}\right)\left(i \not D_{\Theta}+M_{\Theta}\right)
$$

and a helicity-odd contribution $T_{-}$which contains no quadratic divergences. We will therefore neglect this contribution in what follows.

If we were to explicitly evaluate the quantities in (19), using an ultraviolet cut-off $\Lambda$ for the momentum integration in

$$
\int d^{4} x d^{4} p \operatorname{STr} \ln \left(p^{2}+H\right)
$$

we would obtain the quadratic divergence

$$
\mathcal{L}_{1}^{\mathrm{QUAD}}=\sqrt{g} \frac{\Lambda^{2}}{32 \pi^{2}} \mathrm{~S} \operatorname{Tr} H
$$

where the supertrace includes a trace over bosonic, fermionic, ghost and ghostino degrees of freedom. As we will be ultimately interested in effective Lagrangians describing superstring theories, we allow 
for noncanonical gauge field kinetic energy by coupling the Yang-Mills sector of the theory to a holomorphic function of chiral multiplets via the gauge kinetic function $f_{a b}=\delta_{a b} f$ with $f=x+i y$. Then the quadratically divergent contributions from the (gauge fixed) gravity sector, the $N_{\chi}$ chiral multiplets and the (gauge fixed) Yang-Mills sector of internal symmetry dimension $N_{G}$ are

$$
\begin{aligned}
\mathrm{S} \operatorname{Tr} H_{\text {grav }} & =-10 V-2 e^{-K} A \bar{A}+\frac{7}{2} r+4 K_{i \bar{m}} \mathcal{D}_{\mu} z^{i} \mathcal{D}^{\mu} \bar{z}^{\bar{m}}-\frac{x}{2} F_{\mu \nu}^{a} F_{a}^{\mu \nu}-\frac{f_{i} \bar{f}^{i}}{2 x^{2}} \mathcal{D}, \\
\mathrm{STr} H_{\chi} & =2 N_{\chi}\left(\widehat{V}+e^{-K} A \bar{A}-\frac{1}{4} r\right)+\frac{f_{i} \bar{f}^{i}}{2 x^{2}} \mathcal{D}+\frac{2}{x} \mathcal{D}_{a} D_{i}\left(T^{a} z\right)^{i}-2 R_{i \bar{m}}\left(e^{-K} \bar{A}^{i} A^{\bar{m}}+\mathcal{D}_{\mu} z^{i} \mathcal{D}^{\mu} \bar{z}^{\bar{m}}\right), \\
\mathrm{STr} H_{\mathrm{YM}} & =\frac{N_{G}}{2} r+\frac{1}{x} \mathcal{D}_{a} \mathcal{D}^{a}+\frac{x}{2} F_{\mu \nu}^{a} F_{a}^{\mu \nu}-\frac{N_{G}}{2 x^{2}} e^{-K} f_{i} \bar{f}^{j} A_{j} \bar{A}^{i}-\frac{N_{G}}{2 x^{2}}\left(\partial_{\mu} x \partial^{\mu} x+\partial_{\mu} y \partial^{\mu} y\right) .
\end{aligned}
$$

The tree-level scalar potential is given by $V=\widehat{V}+\mathcal{D}$ with

$$
\widehat{V}=e^{-K}\left(A_{i} \bar{A}^{i}-3 A \bar{A}\right) ; \quad \mathcal{D}=\frac{1}{2 x} \mathcal{D}_{a} \mathcal{D}^{a}, \quad \mathcal{D}_{a}=K_{i}\left(T^{a} z\right)^{i} .
$$

Combining all of these we get the total light field contribution

$$
\begin{aligned}
\mathrm{S} \operatorname{Tr} H= & -\left(N_{\chi}-N_{G}-7\right) \frac{r}{2}+2\left(N_{\chi}-5\right) V-\left(N_{\chi}-1\right) \frac{1}{x} \mathcal{D}_{a} \mathcal{D}^{a}+\frac{2}{x} \mathcal{D}_{a} D_{i}\left(T^{a} z\right)^{i} \\
& +2\left(N_{\chi}-1\right) e^{-K} A \bar{A}-2\left(R_{i \bar{m}}-2 K_{i \bar{m}}\right) \mathcal{D}_{\mu} z^{i} \mathcal{D}^{\mu} \bar{z}^{\bar{m}}-2 R_{i \bar{m}} e^{-K} \bar{A}^{i} A^{\bar{m}} \\
& -\frac{N_{G}}{2 x^{2}} e^{-K} f_{i} \bar{f}^{j} A_{j} \bar{A}^{i}-\frac{N_{G}}{2 x^{2}}\left(\partial_{\mu} x \partial^{\mu} x+\partial_{\mu} y \partial^{\mu} y\right),
\end{aligned}
$$

which we can write in a slightly more compact and suggestive manner ${ }^{5}$ by writing $f_{i} \partial_{\mu} z^{i}=i \partial_{\mu} x+$ $i \partial_{\mu} y$ and by noting that $F^{i}=-e^{-K / 2} \bar{A}^{i}=-e^{-K / 2} K^{i \bar{m}} \bar{A}_{\bar{m}}$ :

$$
\begin{aligned}
\mathrm{S} \operatorname{Tr} H= & -\left(N_{\chi}-N_{G}-7\right) \frac{r}{2}-8 \mathcal{D}+\left(28-4 N_{\chi}\right) e^{-K} A \bar{A}+2 K_{i \bar{m}}\left[2 \mathcal{D}_{\mu} z^{i} \mathcal{D}^{\mu} \bar{z}^{\bar{m}}+\left(N_{\chi}-5\right) F^{i} \bar{F}^{\bar{m}}\right] \\
& -2\left(\frac{N_{G}}{4 x^{2}} f_{i} \bar{f}_{\bar{m}}+R_{i \bar{m}}\right)\left(\mathcal{D}_{\mu} z^{i} \mathcal{D}^{\mu} \bar{z}^{\bar{m}}+F^{i} \bar{F}^{\bar{m}}\right)+\frac{2}{x} \mathcal{D}_{a} D_{i}\left(T^{a} z\right)^{i} .
\end{aligned}
$$

Note that by making the following identifications

$$
\left\langle e^{-K} A \bar{A}\right\rangle=m_{3 / 2}^{2} ; \quad\left\langle\frac{f_{i} \bar{f}_{\bar{m}}}{4 x^{2}} F^{i} \bar{F}^{\bar{m}}\right\rangle=m_{1 / 2}^{2} ; \quad\left\langle K_{j}^{k} e^{-K} A \bar{A}-R_{j i \bar{m}}^{k} F^{i} \bar{F}^{\bar{m}}\right\rangle=\left(m^{2}\right)_{j}^{k}
$$

we recognize a contribution to the vacuum energy analogous to that given in (6).

\footnotetext{
${ }^{5}$ In arriving at (25) and (26) we have corrected some errors in expressions found in [18, 26]. These corrections are summarized in Appendix B and can also be found in [32 33].
} 
A subset of these terms were considered in [10] where the potential impact on soft terms was investigated. Yet the one-loop Lagrangian (22), with traces given by (23), is not yet in a suitable form for extracting information on the resulting low-energy theory. Most obviously, the loop contribution to the Einstein term in the gravity action

$$
\mathcal{L}=\left[1-\frac{\Lambda^{2}}{32 \pi^{2}}\left(N_{\chi}-N_{G}-7\right)\right] \frac{r}{2}+\ldots
$$

indicates that the theory cannot be consistently expanded about a flat metric until a Weyl rescaling is performed to render this term canonical. But this straightforward concern is not the only one. We expect our supergravity theory to represent merely an effective Lagrangian, completed at some high energy scale by an underlying - and presumably finite - theory, such as string theory. Thus, the apparent divergences must be rendered finite by the inclusion of an appropriate regulating sector (with typical mass scale $\mu$ as in (7)) before any low-energy phenomenology can be computed [23]. Low-energy results will depend on the $\eta_{S}$ and this can only be obtained by employing a regularization scheme consistent with local supersymmetry (as well as the known symmetries of the underlying theory). That is, the coefficients of the quadratically divergent terms are unreliable in the absence of a manifestly supersymmetric regularization procedure.

\subsection{Rendering the Divergence Finite à la Pauli-Villars}

We now return to (19) but this time we include in the traces the contributions from a Pauli-Villars regulating sector [26, 32, 33. We separate the effective PV mass into two contributions

$$
M_{\mathrm{PV}}^{2}=H_{\mathrm{PV}}(\phi)+\left(\begin{array}{cc}
\mu^{2} & \nu \\
\nu^{\dagger} & \mu^{2}
\end{array}\right) \equiv H_{\mathrm{PV}}+\mu^{2}+\nu .
$$

The first contribution, $H_{\mathrm{PV}}(\phi)$, is the analog to the objects labeled $H$ for the light fields of the theory. It is a field-dependent quantity for which any mass scale is only implicit, via the vev of one or more light fields. But in addition we have true supersymmetric mass terms (i.e. terms that arise from superpotential couplings) that we introduce as well. ${ }^{6}$ We assume these masses to represent the scale of the UV-completion of the supergravity theory, and thus $|\nu|^{2} \sim \mu^{2} \gg H_{\mathrm{PV}} \sim H$.

\footnotetext{
${ }^{6}$ While these terms have explicit mass scales associated with them, they may still retain some residual fielddependence. For example, maintenance of modular invariance in the regulated low-energy theory will often require that these mass terms have some Kähler modulus dependence.
} 
Combining the field-dependent contributions of the light and heavy (PV) fields, we define the matrix $H^{\prime}=H+H^{\mathrm{PV}}$ and now re-express the integral (21) with these contributions included

$$
S_{1}^{\mathrm{QUAD}}=\frac{1}{32 \pi^{2}} \int \mathrm{d}^{4} x \mathrm{~d}^{4} p \sqrt{g} \mathrm{~S} \operatorname{Tr} \ln \left(p^{2}+H^{\prime}+\mu^{2}+\nu\right) .
$$

Expanding the logarithms in (30) in powers of $\left(H^{\prime}+\nu\right) /\left(p^{2}+\mu^{2}\right)$, and using the fact that $H^{\prime} \ll$ $\nu \ll \mu^{2}$ with $\operatorname{Tr} \nu=0$, the momentum integration can be performed. Requiring that the coefficient of the $\Lambda^{2}$ term (the quadratic divergence) vanishes implies that

$$
\mathrm{S} \operatorname{Tr}\left(\mu^{2}\right)=\mathrm{S} \operatorname{Tr} H^{\prime}=0
$$

while requiring the coefficient of the $\ln \Lambda^{2}$ term (the logarithmic divergence) vanishes implies additional conditions [26, 32, 33]. The vanishing of $\mathrm{S} \operatorname{Tr}\left(\mu^{2 n}\right)$ is automatically ensured by supersymmetry. Once all conditions are satisfied, the momentum integration is rendered finite and the resulting one loop contribution is now proportional to the square of the explicit PV masses in (29)

$$
S_{1}^{\mathrm{QUAD}}=-\int \frac{\mathrm{d}^{4} x}{64 \pi^{2}} \sqrt{g} \mathrm{~S} \operatorname{Tr}\left[\left(2 \mu^{2} H^{\prime}+\nu^{2}\right) \ln \mu^{2}\right]+\mathcal{O}\left(\ln \mu^{2}\right) .
$$

To obtain the explicit forms of the matrices $H_{\mathrm{PV}}, \mu$ and $\nu$ we must specify the Pauli-Villars field content. The regulation of matter and Yang-Mills loop contributions to the matter wave function renormalization requires the introduction of PV chiral superfields $\Phi^{P}=\Phi_{\alpha}^{I}, \widehat{\Phi}_{\alpha}^{I}$, $\Phi^{a}$, which transform according to the chiral matter, anti-chiral matter and adjoint representations of the gauge group and have signatures $\eta_{p}^{P}=-1,+1,+1$, respectively for modes $p$ labeled collectively by $P$. Note that full regulation of the theory requires $\alpha$ copies of chiral fields with the same gauge quantum numbers as the light fields $Z^{i}$. These fields couple to the light fields through the superpotential

$$
W\left(\Phi^{P}, Z^{i}\right)=\frac{1}{2} \sum_{\alpha} W_{i j}\left(Z^{k}\right) \Phi_{\alpha}^{I} \Phi_{\alpha}^{J}+\sqrt{2} \sum_{\alpha} \Phi^{a} \widehat{\Phi}_{\alpha}^{I}\left(T_{a} Z\right)_{i}+\cdots
$$

where $T_{a}$ is a generator of the gauge group $\mathcal{G}_{a}$. The Kähler potential for these fields can be written in the general form

$$
K\left(\Phi^{P}, \bar{\Phi}^{P}\right)=\kappa_{I \bar{M}}^{\Phi} \Phi_{\alpha}^{I} \bar{\Phi}_{\alpha}^{\bar{M}}+\widehat{\kappa}_{I \bar{M}}^{\Phi} \widehat{\Phi}_{\alpha}^{I} \widehat{\bar{\Phi}}_{\alpha}^{\bar{M}}+\kappa_{a}^{\Phi}\left|\Phi^{a}\right|^{2}
$$

where the functions $\kappa^{P}$ are a priori functions of the hidden sector (moduli) fields. The PV mass for each superfield $\Phi^{P}$ is generated by coupling it to another field $\Pi^{P}=\left(\Pi^{I}, \widehat{\Pi}^{I}, \Pi^{a}\right)$ in the representation of the gauge group conjugate to that of $\Phi^{P}$ through a superpotential term

$$
W_{m}=\sum_{p} \mu_{P Q}\left(Z^{n}\right) \Phi^{P} \Pi^{Q}
$$


where $\mu_{P Q}\left(Z^{n}\right)$ can in general be a holomorphic function of the light superfields. This is the origin of the explicit $\mu$ and $\nu$-dependent mass terms in (29).

These regulator fields $\Phi^{P}$ must be introduced in such a way as to cancel the quadratic divergences of the light field loops - and thus their Kähler potential is determined relative to that of the fields which they regulate. Specifically we have

$$
\begin{cases}\Phi^{I}: & \kappa_{I \bar{M}}^{\Phi}=\kappa_{i \bar{m}}=K_{i \bar{m}} \\ \widehat{\Phi}^{I}: & \hat{\kappa}_{I \bar{M}}^{\Phi}=\kappa_{i \bar{m}}^{-1}=K^{i \bar{m}} \\ \Phi^{a}: & \kappa_{a}^{\Phi}=g_{a}^{-2} e^{K}\end{cases}
$$

where $g_{a}$ is the (possibly field-dependent) gauge coupling constant for the gauge subgroup $\mathcal{G}_{a}$. There is no similar constraint on the Kähler potential for the fields $\Pi^{P}$, but this uncertainty plays no significant role in an examination of the contributions to the scalar potential in which we are interested. ${ }^{7}$ For concreteness, in the following we set

$$
\begin{cases}\Pi^{I}: & \kappa_{I \bar{M}}^{\Pi}=\delta_{i \bar{m}} e^{\alpha^{I} K} \\ \widehat{\Pi}^{I}: & \hat{\kappa}_{I \bar{M}}^{\Pi}=K_{i \bar{m}} e^{\hat{\alpha}^{I} K} \\ \Pi^{a}: & \kappa_{a}^{\Pi}=g_{a}^{2} e^{\left(\alpha^{a}-1\right) K} .\end{cases}
$$

To regulate the quadratically divergent terms arising from the non-canonical nature of the gauge kinetic energy - such as the last term in (25) - we will here take a very simple PV sector in which we add $N_{G}$ chiral multiplets $\varphi^{\alpha}$ for each gauge group factor, with a universal Kähler potential coupling

$$
K(\varphi, \bar{\varphi})=\kappa_{\alpha}^{\varphi}\left|\varphi^{\alpha}\right|^{2} ; \quad \kappa_{\alpha}^{\varphi}=(f+\bar{f})=2 g_{\mathrm{STR}}^{-2},
$$

where $f=x+i y$ is the field-dependent coefficient of the gauge kinetic function introduced earlier, and $g_{a}=g_{\mathrm{STR}}$ at the string scale. The supersymmetric mass term for this set of fields arises from the following term in the superpotential

$$
W\left(\varphi^{\alpha}\right)=\frac{1}{2} \sum_{\alpha} \mu_{\varphi}^{\alpha}\left(\varphi^{\alpha}\right)^{2}
$$

If we were to consider logarithmic divergences, including dilaton-dependent terms that arise from gauge loops, we would introduce a different set of chiral superfields with different Kähler potential couplings [33], but this is sufficient for our purposes.

\footnotetext{
${ }^{7}$ See, for example, 34, 35] for the impact of these fields on logarithmic contributions to the one-loop scalar potential.
} 
In addition to these chiral superfields, the regulation of the term in (26) proportional to the curvature $r$ requires the introduction of $U(1)_{b}$ gauge multiplets $W^{b}$ with signature $\eta^{b}$. As these regulator fields must have a supersymmetric mass, we must introduce a chiral superfield $\Phi^{b}=e^{\theta_{b}}$, with the same signature, which will eventually form a massive vector supermultiplet with the $W^{b}$ fields. The Kähler potential for these chiral fields is given by

$$
K(\theta, \bar{\theta})=\frac{1}{2} \sum_{b} \nu_{b} e^{\alpha_{b} K}\left(\theta_{b}+\bar{\theta}_{b}\right)^{2} .
$$

This Kähler potential has an invariance under this $U(1)$ for which the field $\Phi^{b}$ has charge $q_{b} \delta_{b c}$ and where $\delta_{c} \theta_{b}=i q_{b} \delta_{b c}$. The corresponding D-term

$$
\mathcal{D}(\theta, \bar{\theta})=\frac{1}{x_{b}} \mathcal{D}_{b} \mathcal{D}^{b} ; \quad \mathcal{D}_{b}=\sum_{c} K_{c} \delta_{b} \theta^{c}=i\left(\theta^{b}+\bar{\theta}^{b}\right) q_{b} e^{\alpha_{b} K} \nu_{b}
$$

vanishes in the background (where we set all Pauli-Villars fields to zero), but the combination $\left(\theta^{b}+\bar{\theta}^{b}\right) / \sqrt{2}$ acquires a supersymmetric squared mass

$$
\mu_{b}^{2}=\frac{1}{2 x_{b}} q_{b}^{2} e^{\alpha_{b}^{\theta} K} \nu_{b}
$$

which is equal to the mass of the vector superfields $W_{b}$, with which it forms a massive vector multiplet in accordance with the Higgs effect. ${ }^{8}$

Having collected all the elements we need for regulating the quadratic divergences we can compute the Pauli-Villars contribution to the supertraces, and thus $H^{\prime}=H+H^{\mathrm{PV}}$ :

$$
\begin{aligned}
\mathrm{S} \operatorname{Tr} H^{\prime}= & 2 \widehat{V}\left[N_{\chi}\left(1+\sum_{\alpha} \eta_{\alpha}^{I}\right)+\sum_{P} \eta_{P}\left(1-\alpha_{P}\right)+\sum_{b} \eta_{b}\left(1-\alpha_{b}\right)-5\right] \\
& +2 e^{-K} A \bar{A}\left[N_{\chi}\left(1+\sum_{\alpha} \eta_{\alpha}^{I}\right)+\sum_{P} \eta_{P}\left(1-3 \alpha_{P}\right)+\sum_{b} \eta_{b}\left(1-3 \alpha_{b}\right)-1\right] \\
& -\frac{r}{2}\left[N_{\chi}\left(1+\sum_{\alpha} \eta_{\alpha}^{I}\right)+\sum_{P} \eta_{P}-7-N_{G}\right]-2 R_{i \bar{m}}\left(e^{-K} \bar{A}^{i} A^{\bar{m}}+\mathcal{D}_{\mu} z^{i} \mathcal{D}^{\mu} \bar{z}^{\bar{m}}\right)\left(1+\sum_{\alpha} \eta_{\alpha}^{I}\right) \\
& +2\left(K_{i \bar{m}} \mathcal{D}_{\mu} z^{i} \mathcal{D}^{\mu} \bar{z}^{\bar{m}}-2 \mathcal{D}\right)\left(2-\sum_{P} \eta_{P} \alpha_{P}-\sum_{b} \eta_{b} \alpha_{b}\right) \\
& +\left(\sum_{\alpha} \eta_{\alpha}^{\varphi}-N_{G}\right)\left[\frac{1}{2 x^{2}} f_{i} \bar{f}_{\bar{m}} \bar{F}^{i} F^{\bar{m}}+\frac{1}{2 x^{2}}\left(\partial_{\mu} x \partial^{\mu} x+\partial_{\mu} y \partial^{\mu} y\right)\right]
\end{aligned}
$$

\footnotetext{
${ }^{8} x_{b}=\operatorname{Re} f_{b}(z)$, where $f_{b}(Z)$ is the gauge kinetic function for $W^{b}$. Regulation of logarithmic divergences from both dilaton and gravity loops requires fields with both $f_{b}(Z)=$ constant $\times S$ and $f_{b}(Z)=$ constant. This in unimportant here and for simplicity we take $f_{b}(Z)=$ constant.
} 
where the summation over the index $P$ is a shorthand for summing over all of the fields $\Phi^{a}, \widehat{\Phi}^{I}$, $\Pi^{A}$ and $\varphi^{\alpha}$. Note that $\alpha_{P}=0$ when $P$ represents the field $\varphi^{\alpha}$. Now given that (43) is the coefficient of the quadratic divergence in (32) it is natural to insist that each term in the expression be separately vanishing, as required by (31). That would imply the following relations among signatures and Kähler factors $\alpha$ :

$$
0=1+\sum_{\alpha} \eta_{\alpha}^{I}=\sum_{P} \eta_{P}+\sum_{b} \eta_{b}-7=\sum_{P} \eta_{P}-7-N_{G}=2-\sum_{P} \eta_{P} \alpha_{P}-\sum_{b} \eta_{b} \alpha_{b}=N_{G}-\sum_{\alpha} \eta_{\alpha}^{\varphi}
$$

This is certainly reasonable in the case where one considers only the quadratic divergences of the theory, as in [26], since an off-shell regularization is possible in this case. That is to say, the theory can be regularized even in the case where the Einstein term is not canonically normalized, as is clearly the case for the curvature term in (43). However, in a complete treatment that includes the logarithmic divergences it is necessary to do a Weyl rescaling at this stage prior to imposing the constraint of (31). More specifically, a Weyl transformation removes a term proportional to the linear combination $\frac{1}{2} r+K_{i \bar{m}} \mathcal{D}_{\mu} z^{i} \mathcal{D}^{\mu} \bar{z}^{\bar{m}}-2 V$, which vanishes on shell due to the graviton equations of motion. Thus we are only requiring on-shell finiteness. Of course, in this case the vanishing of the logarithmic divergences will impose additional constraints on the signatures $\eta$ and Kähler factors $\alpha$.

Having imposed these constraints, we are now in a position to evaluate the terms quadratic in the Pauli-Villars masses in (32). We can simplify the expressions by factoring out of the mass terms any dependence on light fields via $\mu_{p}=\beta_{p} \mu_{P}(z)$ and $\nu_{b}=x_{b}\left(\beta_{b}^{\theta}\right)^{2}\left|\mu_{\theta}(z)\right|^{2}$. Without loss of generality we will set $q_{b}=1$ in (42) and take $\alpha_{a} \equiv \alpha_{\Phi}, \alpha_{b} \equiv \alpha_{\theta}$ and $\beta_{b} \equiv \beta_{\theta}$ to be independent of $a$ and $b$, respectively. Then the relevant terms are

$$
\begin{aligned}
\mathrm{S} \operatorname{Tr}\left(2 \mu^{2} H^{\prime}+\nu^{2}\right)= & e^{-K} A_{I J} \bar{A}^{I J}\left[K_{i \bar{m}} \mathcal{D}_{\mu} \bar{z}^{\bar{m}} \mathcal{D}^{\mu} z^{i}+3 e^{-K} A \bar{A}\right]+2 e^{-2 K} A_{I J} \bar{A}^{J K} R_{n}^{m}{ }_{n} A_{m} \bar{A}^{n} \\
& +e^{-2 K}\left[A_{k I J} \bar{A}^{I J m} \bar{A}^{k} A_{m}-\left(A_{I j K} \bar{A}^{I K} \bar{A}^{j} A+\text { h.c. }\right)\right]-\frac{e^{-K}}{x} \mathcal{D}_{a}\left(T^{a} z\right)^{i} A_{i J K} \bar{A}^{J K} \\
& +\mathcal{D}_{\mu} \bar{z}^{\bar{m}} \mathcal{D}^{\mu} z^{i} e^{-K}\left(A_{i J K} \bar{A}_{\bar{m}}^{J K}+2 R_{i \bar{m} J}^{K} e^{-K} A_{K L} \bar{A}^{J L}\right) \\
& +4 \sum_{b} e^{\alpha_{b}^{\theta} K}\left|\beta_{b} \mu_{\theta}\right|^{2}\left[\left(\widehat{V}+e^{-K} A \bar{A}\right)-e^{-\left(1+\alpha_{b}^{\theta}\right) K}\left|A-\left(\alpha_{b}^{\theta} K_{\bar{m}}+\partial_{\bar{m}} \ln \bar{\mu}_{\theta}\right) A^{\bar{m}}\right|^{2}\right. \\
& -\mathcal{D}_{\mu} \bar{z}^{\bar{m}} \mathcal{D}^{\mu} z^{i}\left(\alpha_{b}^{\theta} K_{\bar{m}}+\partial_{\bar{m}} \ln \bar{\mu}_{\theta}\right)\left(\alpha_{b}^{\theta} K_{i}+\partial_{i} \ln \bar{\mu}_{\theta}\right) \\
& \left.-\alpha_{b}^{\theta} K_{i \bar{m}}\left(\mathcal{D}_{\mu} \bar{z}^{\bar{m}} \mathcal{D}^{\mu} z^{i}+A^{\bar{m}} \bar{A}^{i}\right)+2 \alpha_{b}^{\theta} \mathcal{D}\right]
\end{aligned}
$$

where we have already inserted the explicit matrix elements for the $\theta_{b}$ fields. The upper-case indices then represent each of the fields we had labeled with $P$ in (43): the $\Phi^{A}$ as well as the $\varphi$ fields. 
Lower-case indices refer to light fields and indices of both types are raised with the inverse metric for the appropriate field. For completeness we collect the relevant matrix elements for all the fields below:

$$
\begin{aligned}
\Phi^{I}, \Pi^{I}: & \sum_{I, J} e^{-K} A_{(I \alpha)(J \alpha)} \bar{A}^{(K \alpha)(J \alpha)}=2 \delta_{I}^{K} \sum_{i=I} e^{K\left(1-\alpha_{\alpha}\right)} K^{i \bar{\imath}}\left|\beta_{\alpha} \mu\right|^{2} \\
& A_{(I \alpha)(J \alpha) k}=\left[\left(1-\alpha_{\alpha}\right) K_{k}-\partial_{k} \ln \mu\right] A_{(I \alpha)(J \alpha)}-\Gamma_{k i}^{\ell} A_{(L \alpha)(J \alpha)} \\
\widehat{\Phi}^{I}, \widehat{\Pi}^{I}: & \sum_{J} e^{-K} A_{(I \alpha)(J \alpha)} \bar{A}^{(K \alpha)(J \alpha)}=2 \delta_{I}^{K} e^{-\widehat{\alpha}_{\alpha} K}\left|\widehat{\beta}_{\alpha} \widehat{\mu}\right|^{2} \\
& A_{(I \alpha)(J \alpha) k}=-\left(\widehat{\alpha}_{\alpha} K_{k}+\partial_{k} \ln \widehat{\mu}\right) A_{(I \alpha)(J \alpha)} \\
\Phi^{a}, \widehat{\Phi^{a}}: & e^{-K} A_{c b} \bar{A}^{a c}=2 \delta_{b}^{a} e^{-\alpha_{a}^{\Phi} K}\left|\beta_{a}^{\Phi} \mu_{\Phi}\right|^{2} \\
& A_{a b i}=-\left(\alpha_{a}^{\Phi} K_{i}+\partial_{i} \ln \mu_{\Phi}\right) A_{a b} \\
\varphi^{\alpha}: & e^{-K} A_{\gamma \beta} \bar{A}^{\alpha \gamma}=\frac{e^{K}}{4 x^{2}} \delta_{\beta}^{\alpha}\left|\beta_{\alpha}^{\varphi} \mu_{\varphi}\right|^{2} \\
& A_{\alpha \beta i}=\left[K_{i}-\frac{f_{i}}{x}-\partial_{i} \ln \mu_{\varphi}\right] A_{\alpha \beta} \\
\theta^{b}: & \frac{1}{x_{b}} \sum_{c, d} \delta^{b} \theta^{d} K_{d \bar{c}} \delta_{b} \bar{\theta}^{\bar{c}}=e^{\alpha_{b}^{\theta} K}\left|\beta_{b}^{\theta} \mu_{\theta}\right|^{2} \\
& A_{b c}=\nu_{b} e^{\alpha_{b}^{\theta} K} A_{c}^{\bar{b}}=\nu_{b} e^{\alpha_{b}^{\theta} K}\left[A-\left(\alpha_{b}^{\theta} K_{\bar{m}}+\partial_{\bar{m}} \ln \bar{\mu}_{\theta}\right) A^{\bar{m}}\right] \delta_{b c} .
\end{aligned}
$$

The relevant terms for the scalar reparameterization connection $\Gamma$ and the associated Riemann tensor $R$ are given by

$$
\begin{aligned}
\left(\Gamma_{\Phi}\right)_{b k}^{a} & =-\left(\Gamma_{\Pi}\right)_{b k}^{a}+\alpha_{a}^{\Phi} \delta_{b}^{a} K_{k}=\delta_{b}^{a}\left(K_{k}-\partial_{k} \ln g_{a}\right) \\
\left(R_{\Phi}\right)_{b k \bar{m}}^{a} & =-\left(R_{\Pi}\right)_{b k \bar{m}}^{a}+\alpha_{a}^{\Phi} \delta_{b}^{a} K_{k \bar{m}}=\delta_{b}^{a}\left(K_{k}-\partial_{\bar{m}} \partial_{k} \ln g_{a}\right) \\
\left(\Gamma_{\Phi}\right)_{(J \beta), k}^{(I \alpha)} & =-\left(\Gamma_{\widehat{\Phi}}\right)_{(I \beta), k}^{(J \alpha)}=\left(\Gamma_{\widehat{\Pi}}\right)_{(J \beta), k}^{(I \alpha)}-\delta_{\beta}^{\alpha} \delta_{J}^{I} \hat{\alpha}_{\beta} K_{k}=\delta_{\beta}^{\alpha} \Gamma_{j k}^{i}, \\
\left(R_{\Phi}\right)_{(J \beta), k \bar{m}}^{(I \alpha)} & =-\left(R_{\widehat{\Phi}}\right)_{(I \beta), k \bar{m}}^{(J \alpha)}=\left(R_{\widehat{\Pi}}\right)_{(J \beta), k \bar{m}}^{(I \alpha)}-\delta_{\beta}^{\alpha} \delta_{J}^{I} \hat{\alpha}_{\beta} K_{k \bar{m}}=\delta_{\beta}^{\alpha} R_{j k \bar{m}}^{i}, \\
\left(\Gamma_{\Pi}\right)_{(J \beta), k}^{(I \alpha)} & =\delta_{\beta}^{\alpha} \delta_{J}^{I} \alpha_{\alpha} K_{k}, \quad\left(R_{\Pi}\right)_{(J \beta), k \bar{m}}^{(I \alpha)}=\delta_{\beta}^{\alpha} \delta_{J}^{I} \alpha_{\alpha} K_{k \bar{m}}, \\
\left(\Gamma_{\varphi}\right)_{\alpha i}^{\beta} & =\delta_{\alpha}^{\beta} \frac{f_{i}}{2 x}, \quad\left(R_{\varphi}\right)_{\alpha i \bar{m}}^{\beta}=-\delta_{\alpha}^{\beta} \frac{f_{i} \bar{f}_{\bar{m}}}{4 x^{2}}, \\
\left(\Gamma_{\theta}\right)_{i b}^{c} & =\delta_{b}^{c}\left(\alpha_{b}^{\theta} K_{i}+\partial_{i} \ln \mu_{\theta}\right), \\
\left(R_{\theta}\right)_{b \overline{d i} \bar{m}} & =-\delta_{b d} e^{\alpha_{b}^{\theta} K} \nu_{b} \alpha_{b}^{\theta} e^{\alpha_{b} \theta K} K_{i \bar{m}}=-\delta_{d c} e^{\alpha_{b}^{\theta} K} \nu_{b} R_{b i \bar{m}}^{c}
\end{aligned}
$$




\subsection{Renormalization of the Kähler Potential}

These, then, are the terms quadratically dependent on the (presumably large) cut-off scale $\mu_{\mathrm{PV}}$. We now have the necessary ingredients to study the phenomenological implications of these terms. Before turning our attention there, however, it is instructive to consider how these terms can be grouped into a renormalization of the space-time metric and the Kähler potential itself. Let us define the (field-dependent) effective cut-offs in terms of the Pauli-Villars mass terms in the following way

$$
\begin{aligned}
\Phi^{I}, \Pi^{I} & : e^{\left(1-\alpha_{\alpha}\right) K} K^{i \bar{\imath}}\left|\beta_{\alpha} \mu\right|^{2} \equiv\left(\beta_{\alpha}\right)^{2} \Lambda_{\alpha}^{2} \\
\widehat{\Phi}^{I}, \widehat{\Pi}^{I} & : 2 e^{-\hat{\alpha}_{\alpha} K}\left|\hat{\beta}_{\alpha} \hat{\mu}\right|^{2} \equiv\left(\hat{\beta}_{\alpha}\right)^{2} \widehat{\Lambda}_{\alpha}^{2} \\
\Phi^{a}, \Pi^{a} & : 2 e^{-\alpha_{\alpha}^{\Phi} K}\left|\beta_{\alpha}^{\Phi} \mu_{\Phi}\right|^{2} \equiv\left(\beta_{\alpha}^{\Phi}\right)^{2} \Lambda_{\Phi}^{2} \\
\varphi^{\alpha} & : \frac{e^{K}}{4 x^{2}}\left|\beta_{\alpha}^{\varphi} \mu_{\varphi}\right|^{2} \equiv\left(\beta_{\alpha}^{\varphi}\right)^{2} \Lambda_{\varphi}^{2} \\
\theta^{b} & : 2 e^{\alpha_{b}^{\theta} K}\left|\beta_{b}^{\theta} \mu_{\theta}\right|^{2} \equiv\left(\beta_{b}^{\theta}\right)^{2}\left(\Lambda_{b}^{\theta}\right)^{2} .
\end{aligned}
$$

With this compact notation the quadratic dependence on the PV mass can be easily grouped into a correction of the Kähler potential and space-time metric. In other words, defining $S_{0}+S_{1}=$ $\int \mathrm{d}^{4} x\left(\mathcal{L}_{0}+\mathcal{L}_{1}\right)$ we have

$$
\mathcal{L}_{0}\left(g_{\mu \nu}^{0}, K\right)+\mathcal{L}_{1}=\mathcal{L}_{0}\left(g_{\mu \nu}, K+\delta K\right), \quad g_{\mu \nu}=g_{\mu \nu}^{0}(1+\epsilon)
$$

The above expression involves a summation over effective cutoffs $\Lambda_{P}$ that runs over all the heavy PV modes $p$ labeled by $P$

$$
\epsilon=-\sum_{P} \lambda_{P} \zeta_{P}^{\prime} \frac{\Lambda_{P}^{2}}{32 \pi^{2}} ; \quad \delta K=\sum_{P} \lambda_{P} \zeta_{P} \frac{\Lambda_{P}^{2}}{32 \pi^{2}}
$$

where

$$
\lambda_{P}=\sum_{p} \eta_{p}^{P}\left(\beta_{p}^{P}\right)^{2} \ln \left(\beta_{p}^{P}\right)^{2}
$$

sums over the various species within each class, and the coefficients $\zeta$ and $\zeta^{\prime}$ are given by

$$
\begin{aligned}
\Phi^{I} ; \widehat{\Phi}^{I} & : \quad \zeta_{I}=1 ; \quad \zeta_{I}^{\prime}=1 \\
\Phi^{a} & : \quad \zeta_{a}=1 ; \quad \zeta_{a}^{\prime}=1 \\
\varphi^{\alpha} & : \quad \zeta_{\varphi}=1 ; \quad \zeta_{\varphi}^{\prime}=1 \\
\theta^{b} & : \quad \zeta_{b}=-4 ; \quad \zeta_{b}^{\prime}=0 .
\end{aligned}
$$


These are precise expressions for the quantities alluded to in equations (7) in Section (1) above. Let us see how the identification in (49) and (50) comes about through an explicit construction. Define the quantity $\widetilde{K}=K+\delta K$ where

$$
\delta K=\sum_{P} \delta K_{P}, \quad \delta K_{P}=\zeta_{P} \lambda_{P} \frac{\Lambda_{P}^{2}}{32 \pi^{2}}=\frac{1}{32 \pi^{2}} \sum_{p} \zeta_{P} \eta_{p} \ln \left(\beta_{p}^{2}\right) \delta k_{p}
$$

and the field dependence of the cut-offs in (48) is here represented by

$$
\delta k_{p}=\beta_{p}^{2} \Lambda_{P}^{2}
$$

The content of (49) can be expressed as an expansion in $\delta K$ as follows [19]

$$
\begin{aligned}
\frac{1}{\sqrt{g}} \mathcal{L}_{0}(\widetilde{K})= & \frac{1}{\sqrt{g}} \mathcal{L}_{0}(K)-\delta K \widehat{V}+\delta K_{i \bar{\jmath}}\left(\mathcal{D}^{\mu} z^{i} \mathcal{D}_{\mu} \bar{z}^{\bar{\jmath}}+F^{i} \bar{F}^{\bar{\jmath}}\right) \\
& -\left(\delta K_{i}\left[F^{i} m_{3 / 2}+\frac{1}{2 x} \mathcal{D}_{a}(T z)^{i}\right]+\text { h.c. }\right) \\
\equiv & \frac{1}{\sqrt{g}} \mathcal{L}_{0}(K)+\frac{1}{32 \pi^{2}} \sum_{P} \zeta_{P} \sum_{p} \eta_{p} \ln \left(\beta_{p}^{2}\right) \ell_{p} .
\end{aligned}
$$

To determine the values of the various $\ell_{p}$ it is necessary to take covariant derivatives of the $\delta k_{p}$. Using the fact that the metric is covariantly constant: $D_{i} K^{I J}=0$, and the relations

$$
\begin{aligned}
A_{I J k} & =D_{k} A_{I J}=\partial_{k} A_{I J}-\Gamma_{I k}^{L} A_{L J}-\Gamma_{J k}^{L} A_{I L}, \quad D_{k}\left(e^{-K} \bar{A}_{\bar{I} \bar{J}}\right)=0, \\
D_{\bar{m}}\left(e^{-K} D_{k} A_{I J}\right) & =e^{-K}\left(K_{k \bar{m}} A_{I J}-R_{I k \bar{m}}^{L} A_{L J}-R_{J k \bar{m}}^{L} A_{I L}\right),
\end{aligned}
$$

we have for $P \neq \theta$

$$
\begin{aligned}
\delta k_{p} & =\beta_{p}^{2} \Lambda_{P}^{2}=e^{-K} A_{I J} \bar{A}^{I J} \\
D_{k} \delta k^{p} & =\delta k_{k}^{p}=D_{k}\left(e^{-K} A_{I J} \bar{A}^{I J}\right)_{p}=e^{-K}\left(A_{I J k} \bar{A}^{I J}\right)_{p}, \\
D_{\bar{m}} D_{k} \delta k^{p} & =\delta k_{k \bar{m}}^{p}=e^{-K}\left(A_{I J k} \bar{A}_{\bar{m}}^{I J}+K_{k \bar{m}} A_{I J} \bar{A}^{I J}-2 R_{I k \bar{m}}^{L} A_{L J} \bar{A}^{I J}\right)_{p} .
\end{aligned}
$$

From (55) it is clear that the factor $\ell_{p}$ for $P \neq \theta$ is given by

$$
\begin{aligned}
\ell_{p}= & -e^{-K}\left(A_{I J} \bar{A}^{I J}\right)_{p} \widehat{V}+e^{-K}\left[A_{I J k} \bar{A}_{\bar{m}}^{I J}+\left(K_{i \bar{m}} A_{I J} \bar{A}^{I J}+2 R_{k \bar{m} I}^{L} A_{L J} \bar{A}^{I J}\right)\right]_{p}\left(\mathcal{D}^{\mu} z^{k} \mathcal{D}_{\mu} \bar{z}^{\bar{m}}+F^{k} \bar{F}^{\bar{m}}\right) \\
& -\left\{e^{-K}\left(A_{I J k} \bar{A}^{I J}\right)_{p}\left[F^{k} m_{3 / 2}+\frac{1}{2 x} \mathcal{D}_{a}\left(T^{a} z\right)^{k}\right]+\text { h.c. }\right\},
\end{aligned}
$$

which is the same as the first 3 lines of (45) with

$$
\widehat{V}=K_{i \bar{m}} F^{i} \bar{F}^{\bar{m}}-3 m_{3 / 2}^{2}, \quad F^{i}=e^{-K / 2} \bar{A}^{i}, \quad m_{3 / 2}^{2}=e^{-K} A \bar{A} .
$$


The same calculation for $P=\theta$ is only slightly more complicated. In this case

$$
\begin{aligned}
\partial_{k} \delta k^{b} & =\delta k_{k}^{b}=\left(\alpha_{b}^{\theta} K_{k}+\partial_{k} \ln \mu_{\theta}\right) \delta k^{b}=\Gamma_{b k}^{b} \delta k^{b}, \\
\partial_{\bar{m}} \partial_{k} \delta k^{b} & =\delta k_{k \bar{m}}^{b}=\left[\left(\alpha_{b}^{\theta} K_{k}+\partial_{k} \ln \mu_{\theta}\right)\left(\alpha_{b}^{\theta} K_{\bar{m}}+\partial_{\bar{m}} \ln \bar{\mu}_{\theta}\right)+\alpha_{b}^{\theta} K_{k \bar{m}}\right] \delta k^{b} \\
& =\left(R_{b k \bar{m}}^{b}+\Gamma_{b k}^{b} \Gamma_{\bar{b} \bar{m}}^{\bar{b}}\right) \delta k^{b},
\end{aligned}
$$

so that the corresponding $\ell_{b}$ is given by

$$
\begin{aligned}
\ell_{b} / \delta k^{b}= & -\widehat{V}+R_{b k \bar{m}}^{b}\left(\mathcal{D}^{\mu} z^{k} \mathcal{D}_{\mu} \bar{z}^{\bar{m}}+F^{k} \bar{F}^{\bar{m}}\right)+\Gamma_{b k}^{b} \Gamma_{\bar{b} \bar{m}}^{\bar{b}}\left(\mathcal{D}^{\mu} z^{k} \mathcal{D}_{\mu} \bar{z}^{\bar{m}}+F^{k} \bar{F}^{\bar{m}}\right) \\
& -\left\{\Gamma_{b k}^{b}\left[F^{k} m_{3 / 2}+\frac{1}{2} \mathcal{D}_{a}\left(T^{a} z\right)^{k}\right]+\text { h.c. }\right\} .
\end{aligned}
$$

From (48) and the definitions in (46) we have

$$
\delta k^{b}=-\frac{4}{x_{b}} K_{b \bar{b}}\left|\delta_{b} \theta^{b}\right|^{2}
$$

and after some algebra the entries in (61) can be identified with the final three lines of (45).

Finally, as emphasized above, the effective one loop action given in (49) respects supersymmetry only to lowest order in the loop expansion parameter $\epsilon=\hbar / 16 \pi^{2}$. More precisely if $g_{\mu \nu}^{R}$ and $K^{R}=K+\delta K$ are the fully renormalized metric and Kähler potential, then (49) can be written as

$$
\mathcal{L}_{\text {tree }}\left(g^{R}, K^{R}\right)=\mathcal{L}_{\text {tree }}(g, K)+\mathcal{L}_{1-\text { loop }}+O\left(\epsilon \ln \Lambda_{\text {eff }}^{2}\right)+O\left(\epsilon^{2}\right)
$$

where the leading $N_{\chi}$ and $N_{G}$ corrections that give potentially significant contributions to the effective potential at one loop are

$$
\delta K=\frac{1}{32 \pi^{2}}\left[N_{\chi} \Lambda_{\chi}^{2}-4 N_{G} \Lambda_{G}^{2}+O(1) \Lambda_{\text {grav }}^{2}\right]=\frac{\epsilon}{2}\left[N_{\chi} \Lambda_{\chi}^{2}-4 N_{G} \Lambda_{G}^{2}+O(1) \Lambda_{\text {grav }}^{2}\right] .
$$

If the coefficients of the various $\Lambda^{2}$ terms are of order unity then in order to preserve local supersymmetry of the Lagrangian we have to retain all terms that follow from the correction (64) to the Kähler potential; this amounts to summing leading terms in $\left(\epsilon N \Lambda_{\chi}^{2}\right)^{n}$ and $\left(\epsilon N_{G} \Lambda_{G}^{2}\right)^{n}$, with the result of the sum just giving a correction to the Kähler potential as dictated by local supersymmetry. 


\section{Phenomenological Applications in String-Based Models}

In the preceding sections we demonstrated how the leading one-loop correction to the scalar potential, which is quadratic in the cutoff, is obtained. The explicit form of this correction was given in (26) and shown to be of the general form of (6). We then added a supersymmetric regulating sector to the theory in the form of a set of massive Pauli-Villars regulator fields. These add new contributions to the one-loop effective potential, rendering the divergent contributions finite. Formally divergent contributions to the one-loop Lagrangian are now replaced with terms proportional to the square of various field-dependent mass terms. The combined contribution is given in (45), providing an explicit realization of the subtractions mentioned in (7).

While it is possible to work directly with the (regularized) Lagrangian represented by (45), doing so ignores the great power of working with a supersymmetric regulating sector - namely, the ability to treat the quadratically divergent corrections as a renormalization of the Kähler potential itself. This correspondence, as written in (55), was demonstrated in Section 2.3. In this section we will take the renormalized Kähler potential of (53) as our starting point in looking at the phenomenological implications of these corrections on vacuum stability and scalar mass terms. For most of this section we will be interested primarily in those terms that involve fields connected with the gauge-charged chiral matter of the light spectrum. The relevant corrections to the Kähler potential are therefore those of (57), and we will make use of the relations in (56).

\subsection{Vacuum Energy and Scalar Masses}

As a first example, suppose that the effective cut-offs are field-independent constants. In this case the quantum corrected effective potential is just

$$
V_{e f f}=\mathcal{D}+e^{K+\Delta K}\left(F^{i} K_{i \bar{m}} \bar{F}^{\bar{m}}-\frac{1}{3}|M|^{2}\right)_{\text {tree }},
$$

where $M$ is the auxiliary field of supergravity whose vacuum expectation value determines the gravitino mass through the equation of motion $M=-3 e^{K / 2} W$. In particular, if supersymmetry breaking is F-term induced, i.e. $\langle\mathcal{D}\rangle=0$, then the tree level condition $F^{i} K_{i \bar{m}} \bar{F}^{\bar{m}}=\frac{1}{3}|M|^{2}$ for vanishing vacuum energy is unmodified by these quantum corrections. In other words, even after including all quadratically divergent contributions to the one-loop effective potential (with a proper supersymmetric regularization), the vacuum energy will continue to vanish provided $\left\langle V_{\text {tree }}\right\rangle=0$ is satisfied. 
In some circumstances this simplification can be achieved. For example it was shown in 33 that the masses of PV fields that regulate the (logarithmically divergent) untwisted matter loop corrections involving renormalizable couplings can be made constant (and thereby modular invariant). However not all masses can be constant and modular invariant. Regulation of quadratic and logarithmic divergences requires (for example) some masses proportional to $e^{K}$ in the matter + gravity sector and proportional to $g_{\mathrm{STR}}^{2}=(s+\bar{s}) / 2$ in the gauge + dilaton sector. Modular invariance of the one-loop corrected Lagrangian then requires either that terms proportional to $e^{K}$ cancel in the supertrace implicit in (53), or that the mass terms in the PV superpotential have a T-dependence that restores modular invariance; the latter would be interpreted as a parameterization of string loop threshold corrections. ${ }^{9}$ Thus we generally expect some modification of the effective potential for moduli in string-based effective supergravity theories, as was anticipated by 9 .

For concreteness, in the remainder of this section we consider the BGW model [36, 37] for gaugino condensation, in which dilaton stabilization was achieved [38, 39] by invoking nonperturbative string [40, 41] and/or QFT [42, 43] corrections to the dilaton Kähler potential. In order to implement the correct Bianchi identity for the gaugino condensate composite superfield - as well as the Green-Schwarz (GS) anomaly cancellation mechanism - it is much more convenient to use the linear multiplet formulation for the dilaton, as was done in [36, 37. In 35] the results were recast in the more familiar language of the chiral multiplet formalism, with the effective tree level potential below the scale of condensation taking the standard form $V=F^{i} K_{i \bar{m}} \bar{F}^{\bar{m}}-\frac{1}{3} M \bar{M}$ with

$$
M=\frac{1}{2} b_{c} u, \quad F^{S}=-\frac{1}{4} K_{S \bar{S}}^{-1}\left(1-\frac{2}{3} b_{c} K_{S}\right) \bar{u}
$$

where $u$ is the vacuum value of the gaugino condensate. The quantity $b_{c}$ is the beta function coefficient of the condensing gauge group $\mathcal{G}_{c}$ :

$$
b_{c}=\frac{1}{16 \pi^{2}}\left(3 C_{c}-\sum_{i} C_{c}^{i}\right),
$$

where $C_{c}$ and $C_{c}^{i}$ are the quadratic Casimirs in the adjoint and matter representations, respectively, of $\mathcal{G}_{c}$. The BGW model is explicitly modular invariant and the Kähler moduli $T^{i}$ are stabilized at self-dual points with vanishing auxiliary fields: $\left\langle F^{T^{i}}\right\rangle=0$. Supersymmetry breaking is therefore dilaton-dominated and the condition for vanishing vacuum energy at tree level in the effective

\footnotetext{
${ }^{9}$ There is a residual noninvariance associated with terms logarithmic in the PV masses that is canceled by the Green-Schwarz term.
} 
theory is

$$
\left\langle V_{\mathrm{eff}}\right\rangle=\left\langle K_{S \bar{S}}\left|F^{S}\right|^{2}-\frac{1}{3}|M|^{2}\right\rangle=0, \quad\left\langle K_{S \bar{S}}^{-1}\right\rangle=\frac{4 b_{c}^{2}}{3\left(1-\frac{2}{3}\left\langle K_{S}\right\rangle b_{c}\right)^{2}}
$$

Classically,

$$
-2\left\langle K_{S}\right\rangle=2\left\langle K_{S \bar{S}}^{\frac{1}{2}}\right\rangle=2\left\langle(s+\bar{s})^{-1}\right\rangle=g_{\mathrm{STR}}^{2} \approx \frac{1}{2},
$$

where $g_{\mathrm{STR}}$ is the value of the gauge coupling constant at the string scale. The last approximate relation in (69) can be inferred from low energy data extrapolated to high energy scales through renormalization group (RG) evolution.

The model is both phenomenologically 44] and cosmologically [45, 46] viable if $b_{c} \approx .05-.06$, so it is clear that (68) cannot be satisfied without invoking nonperturbative string effects that modify the Kähler potential for the dilaton. The suppression of the vacuum value $\left\langle K_{S \bar{S}}^{-1}\right\rangle$ with respect to its classical value implied by (68) has the effect of enhancing the dilaton mass, which is a welcome feature for modular cosmology. However it also entails a suppression of gaugino masses relative to scalar masses, which increases somewhat the fine tuning problem of MSSM phenomenology [47. The problem is exacerbated [48, 49] when a D-term arising from an anomalous $U(1)$ is included. Except in a class of models in which the preferred vacuum configuration minimizes the number of large vevs $\left\langle\phi^{a}\right\rangle$, a further suppression of the dilaton F-term is required to maintain vanishing vacuum energy at tree level. In this case a large, positive constant value of $\Delta K$ in (65) would be welcome. In addition there are dangerously large and potentially negative D-term contributions to squark and slepton masses unless $-K_{S}$ is considerably larger than its classical value, thereby calling into question the validity of the weak coupling approximation for these models [42, 50].

Now let us consider the possible impact on the dilaton potential of field dependent effective cut-offs of the type discussed above. With the Green-Schwarz term included modular invariance dictates a renormalized Kähler potential of the form

$$
K^{R}=K+\frac{1}{2} \epsilon c_{\chi} f(T, \bar{T}) e^{K}-\frac{4 \epsilon c_{G}}{S+\bar{S}-V_{\mathrm{GS}}}=K+\frac{1}{2} \epsilon c_{\chi} f(T, \bar{T}) e^{K}-2 \epsilon c_{G} g_{s}^{2}(Z, \bar{Z}),
$$

where $V_{\mathrm{GS}}(T+\bar{T})$ is the Green-Schwarz term and $f(T, \bar{T})$ is a function with a well-defined transformation property under modular symmetries that assures modular invariance of the second term. The loop-induced terms proportional to $\epsilon$ may not be negligible if $c_{\chi}, c_{G} \sim N_{\chi}, N_{G}$, respectively. Since the theory is still modular invariant we expect that the moduli are still stabilized at self-dual points, where the additional contributions to $F^{T^{i}}$ induced by these quantum corrections vanish. Setting the T-moduli at their vevs, defining

$$
\tilde{c}_{\chi}=\left\langle f(t, \bar{t}) e^{G}\right\rangle c_{\chi}
$$


and setting the matter fields to zero, the renormalized potential and its $S$-derivatives read

$$
\begin{aligned}
K^{R} & =k+\frac{\epsilon}{2}\left(\tilde{c}_{\chi} e^{k}-4 c_{G} g_{\mathrm{STR}}^{2}\right), \quad k=k\left(2 g_{\mathrm{STR}}^{-2}\right), \quad K_{S}^{R}=K_{S}\left(1+\frac{\epsilon}{2} \tilde{c}_{\chi} e^{k}\right)+\epsilon c_{G} g_{\mathrm{STR}}^{4}, \\
K_{S \bar{S}}^{R} & =K_{S \bar{S}}\left(1+\frac{\epsilon}{2} \tilde{c}_{\chi} e^{k}\right)+\frac{\epsilon}{2}\left(K_{S}^{2} \tilde{c}_{\chi} e^{k}-c_{G} g_{\mathrm{STR}}^{8}\right) .
\end{aligned}
$$

The condition for vanishing vacuum energy is still given by (68) but with the replacement $K \rightarrow$ $K^{R}$. The relevant parameter for particle physics phenomenology is now the vev of $1 / K_{S \bar{S}}^{R}$, which remains strongly suppressed with respect to its classical value since $K_{S}^{R}$ is negative semi-definite. ${ }^{10}$ Therefore the salient phenomenological features of the BGW model are essentially unaffected by these quantum corrections.

On the other hand, could these corrections lessen the need to invoke rather large nonperturbative effects, especially when an anomalous $U(1)$ is incorporated? A large negative value of $c_{G}$ or a large positive value of $\tilde{c}_{\chi}$ would increase $-K_{S}^{R}$ and decrease $1 / K_{S \bar{S}}^{R}$ for fixed $g_{\mathrm{STR}}^{2} \approx 1 / 2$, which is the desired effect. One can reasonably assume that $\left|c_{G}\right| \leq N_{G} \leq 65 \sim 0.4 \epsilon^{-1}$ in typical orbifold compactifications [22], so a significant effect cannot be obtained from the second term in (1701). On the other hand $N_{\chi} \epsilon \sim 2$ for typical orbifolds. Quite generally we have

$$
f(t, \bar{t})=\prod_{i}\left[\left|\eta\left(t^{i}\right)\right|^{4}\left(t^{i}+\bar{t}^{i}\right)\right]^{q_{i}}, \quad\left\langle\left|\eta\left(t^{i}\right)\right|^{4}\left(t^{i}+\bar{t}^{i}\right)\right\rangle \approx 1,
$$

where the last approximate equality holds at a self-dual point. In this case if $\tilde{c}_{\chi} \sim N_{\chi}$ and $e^{k} \sim 1$, it might be possible to somewhat alleviate the above-mentioned difficulties of condensation models.

One would also expect corrections to the Kähler potential to affect the overall size of scalar masses (we will address the off-diagonal structure of these masses in the next subsection). In the condensation models (with or without an anomalous $U(1)$ ) considered here the scalar mass terms in the effective potential arise from derivatives of the Kähler potential, and the Kähler metric factors out of the normalized squared mass if the Kähler potential is of the form

$$
K=k(S, \bar{S})+g(T, \bar{T})+\sum_{a} \kappa_{a}(T, \bar{T})\left|\Phi^{a}\right|^{2}+O\left(\Phi^{3}\right)
$$

If the second term in (70) is present, the Kähler potential is no longer separable as in (74), and the F-term in the potential takes the form (again setting the moduli at their self-dual points)

$$
V_{F}=K_{S \bar{S}}^{R}\left|F^{S}\right|^{2}+K_{a \bar{b}}^{R} F^{a} \bar{F}^{\bar{b}}+\left(K_{S \bar{b}}^{R} F^{S} \bar{F}^{\bar{b}}+\text { h.c. }\right), \quad F^{a}=\frac{b_{c} \bar{u}}{6} K_{R}^{a \bar{b}} K_{\bar{b}}^{R} .
$$

\footnotetext{
${ }^{10}$ The relation $\ell=-K_{S}^{R}$ holds at any given order in perturbation theory, where $\ell$ is the dilaton of the dual linear multiplet formalism.
} 
The wave function normalization factor $\kappa_{a} /\left(1+\frac{1}{2} \epsilon \tilde{c} e^{k}\right)$ again cancels, and - neglecting the third term in (170) - we obtain for the squared mass of the canonically normalized gauge-charged scalar $\phi^{a}$

$$
\begin{aligned}
m_{\phi^{a}}^{2} & =\frac{b_{c}^{2}|u|^{2}}{36}\left[1+\frac{3}{2} \epsilon \tilde{c} e^{k} \frac{1-\frac{2}{3} b_{c} K_{S}^{R}}{K_{S \bar{S}}^{R}}\left(\frac{1-\frac{2}{3} b_{c} K_{S}^{R}}{K_{S \bar{S}}^{R}}-b_{c} K_{S}^{R}\right)\right]+\frac{1}{s} \sum_{b} q_{a}^{b} D_{b} \\
& =\frac{b_{c}^{2}|u|^{2}}{36}\left[1+\frac{2 b_{c}^{3} \epsilon \tilde{c} e^{k}}{1-\frac{2}{3} b_{c} K_{S}^{R}}\left(\frac{4 b_{c}}{3\left(1-\frac{2}{3} b_{c} K_{S}^{R}\right)}-K_{S}^{R}\right)\right]+\frac{1}{s} \sum_{b} q_{a}^{b} D_{b},
\end{aligned}
$$

where in the last expression we used the vacuum condition (68). Even if $\tilde{c}_{\chi} \sim 1$, these corrections are subleading if $b_{c} \ll 1$.

The primary conclusion of this section is that the only practical effect of the leading $N_{\chi}, N_{G}$ quadratic divergences in this particular concrete example is a possible re-interpretation of the modification of the dilaton superpotential, needed for stabilization, in terms of a combination of string nonperturbative effects and quantum field theory perturbative effects, as opposed to only the former. We note the importance of the supersymmetric regularization in allowing an interpretation of the quadratic contributions to the dilaton effective potential in terms of renormalization of the Kähler potential as in (170). This interpretation made it possible to quickly see the muted impact of these terms, even in the case of large $c_{G}$ and $\tilde{c}_{\chi}$.

\subsection{Potential Off-Diagonal Scalar Mass Terms}

To address the question of what constraints are needed to avoid experimentally excluded flavor changing neutral current (FCNC) effects, we first note that the tree potential of an effective supergravity theory includes a term

$$
V_{\text {tree }} \ni e^{K} K_{i} K_{\bar{\jmath}} K^{i \bar{\jmath}}|W|^{2} .
$$

So prior to any discussion of large loop-induced contributions to flavor-changing operators it is necessary to ensure their absence at the tree level. The observed suppression of FCNC effects thus constrains the Kähler potential already at the leading order - to a high degree of accuracy we require that

$$
K_{i} K_{\bar{\jmath}} K^{i \bar{\jmath}} \not \supset\langle f(X, \bar{X})\rangle \phi_{f}^{a} \bar{\phi}_{f^{\prime} \neq f}^{\bar{a}},
$$

where $f, f^{\prime}$ are flavor indices, $a$ is a gauge index, $\phi_{f}^{a}$ any standard model squark or slepton, and $X$ is a singlet of the Standard Model gauge group. For example, in the no-scale models that characterize the untwisted sector of orbifold compactifications, we have

$$
K_{i} K_{\bar{\jmath}} K^{i \bar{\jmath}}=3+K_{S} K_{\bar{S}} K^{S \bar{S}}
$$


which is safe, since $K_{S}$ is a function only of the dilaton. The twisted sector Kähler potential is known only to quadratic order:

$$
K_{T}=\sum_{a} e^{g^{a}(T+\bar{T})}\left|\Phi_{T}^{a}\right|^{2}+O\left(\Phi^{3}\right)
$$

which is flavor diagonal and also safe. The higher order terms in (80) could be problematic if some $\phi^{a}=X^{a}$ have large vevs (i.e. within a few orders of magnitude of the Planck scale). Thus phenomenology requires that we forbid couplings of the form $\phi_{f}^{a} \phi_{f^{\prime} \neq f^{\bar{a}}}^{\overline{a^{\prime}}}\left|\phi_{f^{\prime \prime}}^{a^{\prime}}\right|^{2} X^{b_{1}} \cdots X^{b_{n}}, n \leq N$, where $N$ is chosen sufficiently large to make the contribution $\left\langle X^{b_{1}} \cdots X^{b_{n}}\right\rangle$ to the scalar mass matrix negligible.

The quadratically divergent one-loop corrections generate a term

$$
V_{1-\text { loop }} \ni e^{K} K_{i} K_{\bar{\jmath}} R^{i \bar{\jmath}}|W|^{2}, \quad R^{i \bar{\jmath}}=K^{i \bar{k}} R_{\bar{k} l} K^{k \bar{\jmath}} \text {. }
$$

where $R_{i \bar{\jmath}}$ is the Kähler Ricci tensor. The contribution (81) simply reflects the fact that the leading divergent contribution in a nonlinear sigma model is a correction to the Kähler metric proportional to the Ricci tensor (whence e.g. the requisite Ricci flatness of two dimensional conformal field theories). Since the Ricci tensor involves a sum of Kähler Riemann tensor elements over all chiral degrees of freedom, a large, order $N_{\chi}$, coefficient may be generated. For example, for an untwisted sector $U$ with three untwisted moduli $T^{i}$ and Kähler potential

$$
K^{U}=\sum_{n=1}^{3} K^{n}=-\sum_{n=1}^{3} \ln \left(T^{n}+\bar{T}^{\bar{n}}-\sum_{a=1}^{N_{n}}\left|\Phi_{n}^{a}\right|^{2}\right),
$$

we get

$$
R_{i \bar{\jmath}}^{n}=\left(N_{n}+2\right) K_{i \bar{\jmath}}^{n}
$$

While this contribution is clearly safe, since the Ricci tensor is proportional to the Kähler metric, the condition that the tree potential be FCNC safe does not by itself ensure that (81) is safe in general. For this we require in addition the absence of Kähler potential terms of the form $\phi_{f}^{a} \bar{\phi}_{f^{\prime} \neq f}^{\bar{a}}\left|\phi_{f^{\prime \prime}}^{a^{\prime}}\right|^{4}\left(X^{b}\right)^{n \leq N}$. On the other hand, if the Kähler metric is FCNC safe due to an isometry, the same isometry will protect the Ricci tensor from generating FCNC.

For example, the scalar metric $g_{i j}$ for the effective pion Lagrangian is dictated by chiral $S U(2)_{L} \times$ $S U(2)_{R}$; there is a unique form of the two-derivative coupling:

$$
g_{i j}=\delta_{i j}+\frac{\pi_{i} \pi_{j}}{v^{2}-\pi^{2}},
$$


for a particular choice of field variables. Preservation of this symmetry at the one-loop level assures that $R_{i j} \propto g_{i j}$. Similarly, the kinetic term derived from the Kähler potential (82) possesses an $\prod_{n=1}^{3} S U\left(N_{n}+1,1\right)$ symmetry that is much larger than the $S L(2, R)$ (or possibly $[S L(2, R)]^{3}$ ) T-duality symmetry of the full Lagrangian, and we obtain the result (83). More generally, in effective supergravity from string compactifications there are a number of selection rules and/or symmetries that forbid superpotential couplings that are allowed by gauge invariance and the $\mathrm{T}$ duality invariance group (see for example [51]). The Kähler potential has not been investigated in similar detail, but a priori one would expect an analogous pattern. In the absence of input from string theory one can work backwards and ask: what constraints does phenomenology impose?

We first note that there is a large class of models in which FCNC are suppressed independently of the details of the structure of the Kähler potential, provided the moduli $t^{I}$ are stablized at self dual points. The supersymmetric completion of the potential in any given order in perturbation theory yields (in the absence of D-term contributions) the scalar squared mass matrix

$$
\left(m^{2}\right)_{j}^{i}=\delta_{j}^{i} m_{3 / 2}^{2}-\left\langle\widetilde{R}_{j k \bar{m}}^{i}\right\rangle \widetilde{F}^{k} \widetilde{\bar{F}}^{\bar{m}}
$$

where $\widetilde{R}_{j k \bar{m}}^{i}$ is an element of the Riemann tensor derived from the fully renormalized Kähler metric, and $\widetilde{F^{i}}$ is the auxiliary field for the chiral superfield $\Phi^{i}$, evaluated by its equation of motion using the quantum corrected Lagrangian. Since the latter is perturbatively modular invariant, the Kähler moduli $t^{i}$ are still stabilized at self-dual points with $\left\langle\widetilde{F}^{t^{i}}\right\rangle=0$. Classically we have $R_{b s \bar{s}}^{a}=0$ where the indices $a, b$ refer to gauge-charged fields in the observable sector. This need not be true at the quantum level. For example, if, as suggested in (70), the quantum correction to the Kähler potential includes a term

$$
\Delta K=\frac{1}{32 \pi^{2}} \mathrm{~S} \operatorname{Tr} \Lambda_{\text {eff }}^{2} \ni \frac{c N_{\chi}}{32 \pi^{2}} e^{\alpha K}
$$

we get

$$
\left\langle\widetilde{R}_{b s \bar{s}}^{a}\right\rangle=\delta_{b}^{a} \frac{c N_{\chi}}{32 \pi^{2}} \alpha^{2} e^{\alpha K}\left(K_{s \bar{s}}+\alpha K_{s} K_{\bar{s}}\right)
$$

which is flavor diagonal, and therefore FCNC safe.

To consider more general situations, let us write out the elements of the renormalized Kähler Riemann tensor

$$
\begin{aligned}
\widetilde{R}_{j k \bar{m}}^{i} & =\widetilde{D}_{\bar{m}} \widetilde{\Gamma}_{k j}^{i}=\widetilde{K}^{i \bar{n}} \widetilde{D}_{\bar{m}} \widetilde{\Gamma}_{\bar{n} k j}=\widetilde{K}^{i \bar{n}}\left[\partial_{\bar{m}} \widetilde{\Gamma}_{\bar{n} k j}-\widetilde{\Gamma}_{\bar{m} \bar{n}}^{\bar{r}} \widetilde{\Gamma}_{\bar{r} k j}\right] \\
& =K^{i \bar{n}}\left[D_{\bar{m}} \widetilde{\Gamma}_{\bar{n} k j}+\left(\Gamma_{\bar{m} \bar{n}}^{\bar{r}}-\widetilde{\Gamma}_{\bar{m} \bar{n}}^{\bar{r}}\right) \widetilde{\Gamma}_{\bar{r} k j}\right] \\
\widetilde{\Gamma}_{\bar{n} k j} & =\partial_{k} \widetilde{K}_{\bar{n} j}=\Gamma_{\bar{n} k j}+\partial_{i} \delta K_{\bar{n} j}=\Gamma_{\bar{n} k j}+D_{i} \delta K_{\bar{n} j}+\Gamma_{k j}^{l} \delta K_{\bar{n} l}
\end{aligned}
$$


with the inverse metric $\widetilde{K}^{i \bar{n}}$ given by

$$
\widetilde{K}^{i \bar{n}}=K^{i \bar{n}}-K^{i \bar{p}} \delta K_{\bar{p} l} K^{l \bar{n}}+O\left(\delta K^{2}\right) .
$$

The potentially dangerous terms in (85) can then be extracted from consideration of (88), for which we have

$$
\widetilde{R}_{j k \bar{m}}^{i}=R_{j k \bar{m}}^{i}-K^{i \bar{n}} \delta K_{\bar{n} l} R_{j k \bar{m}}^{l}+K^{i \bar{n}} D_{\bar{m}} D_{k} \delta K_{\bar{n} j}+O\left(\delta K^{2}\right),
$$

and the auxiliary fields for the chiral superfields, given by

$$
\widetilde{F}^{i}=e^{\widetilde{K} / 2} \widetilde{K}^{i \bar{\jmath}}\left(\bar{W}_{\bar{\jmath}}+\bar{W} \widetilde{K}_{\bar{\jmath}}\right)=F^{i}\left(1+\frac{\delta K}{2}\right)-K^{i \bar{\jmath}} \delta K_{\bar{\jmath} k} F^{k}+e^{K / 2} K^{i \bar{\jmath}} \delta K_{\bar{\jmath}} \bar{W}+O\left(\delta K^{2}\right) .
$$

Then the second term in (85) is given by

$$
\begin{aligned}
\left(m_{R}^{2}\right)_{j}^{i}= & -\widetilde{R}_{j k \bar{m}}^{i} \widetilde{F}^{k} \widetilde{\bar{F}}^{\bar{m}} \\
= & -R_{j k \bar{m}}^{i} F^{k} \bar{F}^{\bar{m}}\left(1+\frac{\delta K}{2}\right)+R_{j}^{i \bar{m}} F^{l} \bar{F}^{\bar{n}} \delta K_{l \bar{m}}+R_{j k}^{i} F^{k} \bar{F}^{\bar{m}} \delta K_{l \bar{m}} \\
& -e^{K / 2}\left(R_{j}^{i \bar{m} \bar{n}} \bar{F}^{\bar{n}} \delta K_{\bar{m}} \bar{W}+R_{j k}^{i} F^{l} \delta K_{l} W\right) \\
& +R_{j k \bar{m}}^{l} F^{k} \bar{F}^{\bar{m}} K^{i \bar{n}} \delta K_{l \bar{n}}-K^{i \bar{n}} F^{k} \bar{F}^{\bar{m}} D_{\bar{m}} D_{j} \delta K_{k \bar{n}}+O\left(\delta K^{2}\right) .
\end{aligned}
$$

Let us investigate the consequences for the following simple Kähler potential

$$
K=g(M, \bar{M})+\sum_{a} f_{a}(M, \bar{M})\left|\Phi^{a}\right|^{2}+O\left(\Phi^{3}\right),
$$

where $M, \bar{M}$ represent chiral superfields of the hidden sector (such as moduli in a string construction) which are SM gauge singlets, and we have chosen a basis in which the term quadratic in the matter fields $\Phi^{a}$ is diagonal. The tree-level contribution to the scalar masses is then

$$
\left\langle R_{b n \bar{m}}^{a} F^{n} \bar{F}^{\bar{m}}\right\rangle=\delta_{b}^{a}\left\langle g_{n \bar{m}}^{a} F^{n} \bar{F}^{\bar{m}}\right\rangle, \quad g_{n \bar{m}}^{a}=f_{a}^{-1} \partial_{\bar{m}} \partial_{n} f_{a}-f_{a}^{-2} \partial_{\bar{m}} f_{a} \partial_{n} f_{a} .
$$

To avoid tree-level FCNC, we require $\left\langle g_{n \bar{m}}^{a} F^{n} \bar{F}^{\bar{m}}\right\rangle$ to be independent of flavor for fixed SM gauge quantum numbers. As a specific example, in string theory with $f_{a}=\left(T^{i}+\bar{T}^{i}\right)^{-q_{a}^{i}}$ we can avoid treelevel FCNC if $\left\langle F^{t^{i}}\right\rangle=0$ or if all quarks with the same quantum numbers have the same modular weights ${ }^{11} q_{a}^{i}: g_{i \bar{j}}^{a}=\delta_{i j} q_{a}^{i}\left(2 \operatorname{Re}^{i}\right)^{-2}$. Assuming tree level FCNC are absent, the only dangerous part

\footnotetext{
${ }^{11}$ Note that two independent conventions for modular weights exist in the literature. In particular, if we use the expression in (93) with $f_{a}=\left(T^{i}+\bar{T}^{i}\right)^{-q_{a}^{i}}$ to serve as the defining property of the integer modular weight, then our convention corresponds to those of [25] 36] 37] 38, while the case $-q_{a}^{i} \rightarrow n_{a}^{i}$ is the convention of [35, 52 [53, 54]. The sign convention on the weights we have chosen has the virtue that the integers $q_{a}^{i}$ will typically be positive.
} 
of (92) is the last line. Using (56), (57), and

$$
\begin{aligned}
D_{l}\left(e^{-K} \bar{A}_{\bar{m}}^{I J}\right)= & e^{-K}\left(K_{l \bar{m}} \bar{A}^{I J} R_{P \bar{m} l}^{I} \bar{A}^{P J}+R_{P \bar{m} l}^{J} \bar{A}^{I P}\right) \\
D_{l} \delta k_{k \bar{m}}= & K_{k \bar{m}} \delta k_{l}+K_{l \bar{m}} \delta k_{k} \\
& +e^{-K}\left[A_{I J k l} \bar{A}_{\bar{m}}^{I J}+2\left(R_{I \bar{m} l}^{P} A_{P J k} \bar{A}^{I J}+[k \leftrightarrow l]\right)+2\left(D_{l} R_{I \bar{m} k}^{P}\right) A_{P J} \bar{A}^{I J}\right] \\
D_{\bar{m}}\left(e^{-K} A_{I J k l}\right)= & e^{-K}\left\{R_{l \bar{m} k}^{n} A_{I J n}+\left[K_{k \bar{m}} A_{I J l}+\left(R_{I \bar{m} k}^{P} A_{P J l}+[I \leftrightarrow J]\right)+(k \leftrightarrow l)\right]\right\} \\
& +e^{-K}\left[A_{P J} D_{l} R_{I \bar{m} k}^{P}+(I \leftrightarrow J)\right]
\end{aligned}
$$

we obtain for the modes $P \neq \theta$

$$
\begin{aligned}
D_{\bar{n}} D_{l} \delta k_{k \bar{m}}= & e^{-K} A_{I J k l} \bar{A}_{\bar{m} \bar{n}}^{I J}+\left[K_{k \bar{m}} \delta k_{l \bar{n}}+(k \leftrightarrow l)+(n \leftrightarrow m)\right]-\left[K_{k \bar{m}} K_{l \bar{n}}+(k \leftrightarrow l)\right] \delta k \\
& +e^{-K}\left[R_{l \bar{n} k}^{p} A_{I J p} \bar{A}_{\bar{m}}^{I J}+2\left(D_{\bar{n}} D_{l} R_{I \bar{m} k}^{P}\right) A_{P J} \bar{A}^{I J}\right] \\
& +2 e^{-K}\left\{\left[\left(D_{l} R_{I \bar{m} k}^{P}\right) A_{P J} \bar{A}_{\bar{n}}^{I J}+(n \leftrightarrow m)\right]+\left[\left(D_{\bar{n}} R_{I \bar{m} l}^{P}\right) A_{P J k} \bar{A}^{I J}+(k \leftrightarrow l)\right]\right\} \\
& +2 e^{-K}\left[R_{I \bar{m} l}^{P} A_{P J k} \bar{A}_{\bar{n}}^{I J}+(n \leftrightarrow m)+(k \leftrightarrow l)\right] \\
& +2 e^{-K}\left[R_{I \bar{m} l}^{Q} R_{Q \bar{n} k}^{P} A_{P J} \bar{A}^{I J}+R_{I \bar{m} l}^{Q} R_{J \bar{n} k}^{P} A_{P Q} \bar{A}^{I J}+(k \leftrightarrow l)\right]
\end{aligned}
$$

Inserting this into the last line of (92) gives

$$
\begin{aligned}
\left(m_{P}^{2}\right)_{b}^{a} \ni- & F^{m} \bar{F}^{\bar{n}}\left\{\delta_{b}^{a} \delta k_{m \bar{n}}+K_{m \bar{n}} K^{a \bar{c}} \delta k_{b \bar{c}}-\delta_{b}^{a} K_{m \bar{n}} \delta k-R_{b m \bar{n}}^{c} K_{a \bar{d}} \delta k_{c \bar{d}}\right. \\
& +2 e^{-K}\left[\left(D_{\bar{n}} D_{b} R_{I m}^{P a}\right) A_{P J} \bar{A}^{I J}+\left(D_{b} R_{I m}^{P a}\right) A_{P J} \bar{A}_{\bar{n}}^{I J}+2\left(D_{\bar{n}} R_{I b}^{P a}\right) A_{P J m} \bar{A}^{I J}\right. \\
& \left.\left.+R_{I b}^{P a} A_{P J m} \bar{A}_{\bar{n}}^{I J}+R_{I b}^{Q a} R_{Q \bar{n} m}^{P} A_{P J} \bar{A}^{I J}+R_{I b}^{Q a} R_{J \bar{n} m}^{P} A_{P Q} \bar{A}^{I J}\right]\right\},
\end{aligned}
$$

where we dropped terms that vanish in the vacuum, and the above "squared masses" have to be put in a weighted sum as in (53) with the appropriate loop factor.

Most of the terms in (97) are in fact proportional to $\delta_{a}^{b}$, so represent corrections to the diagonal elements of the scalar mass matrix. The off-diagonal elements are the result of terms involving the set of PV fields $P=\Phi^{I}$, in which we identify $R_{J k l}^{I} \rightarrow R_{j k l}^{i}$ :

$$
\begin{aligned}
\left(m_{\Phi_{\alpha}^{I}, \Pi_{\alpha}^{I}}^{2}\right)_{b}^{a} \ni & -e^{-K} F^{m} \bar{F}^{\bar{n}}\left[K_{m \bar{n}} R_{i b}^{p a} A_{P J} \bar{A}^{I J}+\left(D_{\bar{n}} D_{b} R_{i m}^{p a}\right) A_{P J} \bar{A}^{I J}\right. \\
& +\left(D_{b} R_{i m}^{p a}\right) A_{P J} \bar{A}_{\bar{n}}^{I J}+\left(D_{\bar{n}} R_{i b}^{p a}\right) A_{P J m} \bar{A}^{I J} \\
& +R_{i b}^{p a} A_{P J m} \bar{A}_{\bar{n}}^{I J}-\alpha R_{i b}^{p a} K_{\bar{n} m} A_{P J} \bar{A}^{I J} \\
& \left.-R_{b m \bar{n}}^{a} A_{I J} A^{I J}-2 R_{b m \bar{n}}^{c} R_{I{ }_{c}}^{P a} A_{P J} A^{I J}\right] .
\end{aligned}
$$


Using the quantities in (46) and (47), along with the identity

$$
2 R_{I \bar{m} k}^{P} A_{P J} \bar{A}^{I J}=\left(R_{k \bar{m}}^{\Phi}+R_{k \bar{m}}^{\Pi}\right)|\beta|^{2} \Lambda^{2}
$$

the potentially dangerous mass terms in (98) can be written as ${ }^{12}$

$$
\begin{aligned}
\left(m_{\Phi_{\alpha}^{I}, \Pi_{\alpha}^{I}}^{2}\right)_{b}^{a} \ni & K^{a \bar{c}} F^{m} \bar{F}^{\bar{n}} \beta_{\alpha}^{2} \Lambda_{\alpha}^{2}\left\{D_{\bar{n}} D_{b} R_{m \bar{c}}+2 R_{d \bar{c}} R_{b m \bar{n}}^{d}\right. \\
& +\left(K_{m \bar{n}}\left(1-\alpha_{\alpha}\right)+\left[\left(1-\alpha_{\alpha}\right) K_{m}-\partial_{m} \ln \mu_{\alpha}\right]\left[\left(1-\alpha_{\alpha}\right) K_{\bar{n}}-\partial_{\bar{n}} \ln \mu_{\alpha}\right]\right) R_{b \bar{c}} \\
& \left.+\left[\left(1-\alpha_{\alpha}\right) K_{\bar{n}}-\partial_{\bar{n}} \ln \mu_{\alpha}\right] D_{b} R_{m \bar{c}}+\left[\left(1-\alpha_{\alpha}\right) K_{m}-\partial_{m} \ln \mu_{\alpha}\right] D_{\bar{n}} R_{b \bar{c}}\right\} .
\end{aligned}
$$

This is the principal result of this section, and we will spend the rest of the section investigating its consequences in a number of simple examples. But first it is instructive to compare the expression in (100) with the analogous expression for the scalar mass in equation (4) of [10. ${ }^{13}$ The two expressions share the same general structure, though the coefficients of the various correction terms differ - presumably since the starting point in [10] was not yet fully supersymmetric. We have checked that (100) has the proper symmetry under interchange of indices implied by its origin from (96).

A more substantive comparison can be made by considering a particular Kähler potential. Take the case of

$$
\begin{gathered}
K=g(M, \bar{M})+\sum_{a} f_{a}(M, \bar{M})\left|\Phi^{a}\right|^{2}+\frac{1}{4} \sum_{a b} X_{a b} f_{a} f_{b}\left|\Phi^{a}\right|^{2}\left|\Phi^{b}\right|^{2}+O\left(|\Phi|^{3}\right), \\
g(M, \bar{M})=-\sum_{i} \ln \left(T^{i}+\bar{T}^{i}\right), \quad f_{a}(M, \bar{M})=\prod_{i}\left(T^{i}+\bar{T}^{i}\right)^{-q_{i}^{a}},
\end{gathered}
$$

which is motivated by modular-invariant effective actions describing the weakly-coupled heterotic string. Then the relevant tensors for the computation of (100) are given by

$$
\begin{aligned}
R_{b \bar{c}} & =\delta_{b c} f_{b}\left(\sum_{a} X_{a b}+\sum_{i} q_{i}^{b}\right), \quad D_{\bar{n}} R_{b \bar{c}}=0, \quad D_{\bar{n}} D_{b} R_{m \bar{c}}=\frac{\delta_{m n}}{2 \operatorname{Re} t^{m}} D_{b} R_{m \bar{c}}, \\
D_{b} R_{m \bar{c}} & =-\delta_{b c} \frac{f_{b}}{2 \operatorname{Re} t^{m}}\left(\sum_{a}\left(q_{m}^{b}+q_{m}^{a}\right) X_{a b}+q_{m}^{b} \sum_{i} q_{i}^{b}\right) .
\end{aligned}
$$

\footnotetext{
${ }^{12}$ In what follows we have a dropped a term $\beta^{2} \Lambda^{2} R_{b m \bar{n}}^{a} F^{m} \bar{F}^{\bar{n}}$ since it will not produce any FCNC contributions if the tree-level masses are FCNC safe.

${ }^{13}$ The analogous expression is equation (6) of the preprint version hep-ph/9709250 of this paper.
} 
First consider the case where $F^{i}=0$ for the Kähler moduli $T^{i}$ and where $F^{s} \bar{F}^{\bar{s}} K_{s \bar{s}}=F^{s} \bar{F}^{\bar{s}} K_{s} K_{\bar{s}}=$ $3 m_{3 / 2}^{2}$. Then assuming $\partial_{s} \mu_{\alpha}=0$ we obtain

$$
\left(m_{\Phi_{\alpha}^{I} \Pi_{\alpha}^{I}}^{2}\right)_{b}^{a}=3 m_{3 / 2}^{2} \beta_{\alpha}^{2} \Lambda_{\alpha}^{2} \delta_{b}^{a}\left(\sum_{c} X_{b c}+\sum_{i} q_{i}^{b}\right)\left(1-\alpha_{\alpha}\right)\left(2-\alpha_{\alpha}\right) .
$$

As was pointed out in [10, where this same Kähler potential (101) was considered, even in this particularly simple case of dilaton-domination (in which tree-level scalar masses are universal and diagonal) there is a potential for sizable FCNCs since the summation in the first term of (104) runs over all fields which participate in the quartic coupling of (101). What was not appreciated in [10] was the fact that the presence of this off-diagonal scalar mass contribution depends on the parameters $\alpha_{\alpha}$, which are determined by Planck-scale physics. In particular, the contribution vanishes completely - independent of the values of the modular weights or the values of $X_{a b}-$ provided $\alpha=1$ or 2 .

For the untwisted sector $\Phi_{i}^{a}$, with Kähler potential given by (82)

$$
K^{U_{i}}=g_{i}-\ln \left(1-e^{g_{i}} \sum_{a}\left|\Phi_{i}^{a}\right|^{2}\right), \quad g_{i}=-\ln \left(T^{i}+\bar{T}^{i}\right),
$$

the expression for $R_{b \bar{c}}$ is given by (83). If the twisted sector Kähler potential is just the quadratic term in (102) then the twisted masses are different from the untwisted ones. If instead the twisted sector has

$$
K^{T}=\sum_{a} e^{\sum_{i} q_{i}^{a} K^{U_{i}}}\left|\Phi_{T}^{a}\right|^{2}
$$

we would instead obtain

$$
\sum_{a} X_{a, b T}=\sum_{i} q_{i}^{a} N_{i}, \quad \sum_{a} X_{a, b i}=2 N_{i}+\sum_{a \in T} q_{i}^{a} .
$$

In orbifold constructions it is common for the quark doublet $Q_{L}$ of the Standard Model to arise in the untwisted sector, though generally at least some of the other Standard Model fields must arise in one or more of the various twisted sectors [22, 51]. Thus we might expect some contributions such as those in (107) to arise. Nevertheless, such terms may be innocuous (quite apart from the issue of the factors of $\alpha$ ) even in cases where $F^{i} \neq 0$ provided all twisted sector SM fields have the same modular weights - a condition that obtains quite often in these models.

Additional terms in (103) can arise if the Kähler potential includes terms in addition to those in (101). For example, we might consider an addition of the form

$$
\Delta K=\left\{\frac{1}{2} Z_{a b} \Phi^{a} \Phi^{b}+\frac{1}{2} H_{a b \bar{c}} \Phi^{a} \Phi^{b} \bar{\Phi}^{\bar{c}}+\frac{1}{3} Z_{a b c} \Phi^{a} \Phi^{b} \Phi^{c}+\ldots\right\}+\text { h.c. }
$$


In fact, such extensions are not uncommon in phenomenological considerations: terms with $Z_{a b} \neq 0$ might be utilized to generate a $\mu$-term via the Giudice-Masiero mechanism [55] while three-field terms with both $H_{a b \bar{c}}$ and $Z_{a b c}$ non-vanishing were utilized in [56] to produce neutrino masses.

In general these terms are constrained by requirements of gauge invariance, which do not affect the case of $X_{a b}$ in (101). For example, a Giudice-Masiero term of $\Delta K=\frac{1}{2} Z(T, \bar{T}) H_{u} H_{d}+$ h.c. can only produce corrections to the mass matrix for the Higgs fields themselves, and there only to the diagonal entries provided the quadratic term in (101) is diagonal in these fields to begin with. This is a general property of additions to the Kähler potential with the form $\Delta K=f(\Phi)+\bar{f}(\bar{\Phi})$.

More dangerous are terms with mixed holomorphicity, such as the case of [56] in which the following was added to the Kähler potential

$$
\Delta K=\left(Z_{1}\right)_{a b} L_{a} H_{u} N_{b}+\left(Z_{2}\right)_{a b} L_{a} H_{d}^{*} N_{b}+\text { h.c. },
$$

where $N$ is a right-handed neutrino superfield, $L$ is the standard lepton doublet of the MSSM and $a, b$ are generation labels. Here we do expect contributions to off-diagonal scalar masses (as well as additional contributions to the diagonal entries) at one loop. For example, we have

$$
\begin{aligned}
R_{L_{a} L_{b}^{*}} & =\sum_{i} q_{b}^{i} f_{L_{b}} \delta_{a b}-\sum_{c} \frac{1}{f_{N_{c}}}\left[\left(Z_{2}\right)_{a c}\left(Z_{2}^{*}\right)_{b c} \frac{1}{f_{H_{d}}}+\left(Z_{1}\right)_{a c}\left(Z_{1}^{*}\right)_{b c} \frac{1}{f_{H_{u}}}\right] \\
R_{N_{a} N_{b}^{*}} & =\sum_{i} q_{b}^{i} f_{N_{b}} \delta_{a b}-\sum_{c} \frac{1}{f_{L_{c}}}\left[\left(Z_{2}\right)_{c a}\left(Z_{2}^{*}\right)_{c b} \frac{1}{f_{H_{d}}}+\left(Z_{1}\right)_{c a}\left(Z_{1}^{*}\right)_{c b} \frac{1}{f_{H_{u}}}\right] .
\end{aligned}
$$

If the coefficients $Z_{1}$ and $Z_{2}$ depend on various moduli (which they will, in general, in realistic string models) then there are additional terms coming from $D_{L_{a}} R_{T_{m} L_{b}^{*}}$ and $D_{N_{a}} R_{T_{m} N_{b}^{*}}$. These terms involve derivatives of the coefficients $Z_{1}$ and $Z_{2}$ and can introduce additional off-diagonal terms if the $F$-terms for the corresponding moduli fields do not vanish in the vacuum.

Though these terms must necessarily be present in this scenario, they need not necessarily be large. The summations in (110) are only over the various species of right-handed neutrino and lepton doublet, respectively, that couple through the terms in (109). While it is not inconceivable that the number of such fields might be greater than three in string-derived models [57, 58, it is unlikely that such sums will generate numbers of $\mathcal{O}(100)$. Nevertheless, the severe constraint on the branching ratio for the rare decay $\mu \rightarrow e \gamma$ of $\operatorname{Br}(\mu \rightarrow e \gamma)<1.2 \times 10^{-11}$ [59, 60] suggests that the size of the element $m_{L_{1} L_{2}^{*}}^{2}$ should be quite small. In particular [4, 61, 62]

$$
\delta_{12}^{L L}=\frac{m_{L_{1} L_{2}^{*}}^{2}}{m_{L_{1} L_{1}^{*}}^{2}} \lesssim 4 \times 10^{-3} ; \quad \text { for } \sqrt{m_{L_{1} L_{1}^{*}}^{2}}=100 \mathrm{GeV}
$$


though the size of the off-diagonal elements can be comparable to the diagonal entries once the typical slepton mass nears $1 \mathrm{TeV}$. For the case of (111) above, insertion of (110) into (100) and summing as in (53) gives an off-diagonal mass contribution of roughly

$$
\delta_{12}^{L L} \simeq \frac{3 N_{\nu_{R}}}{32 \pi^{2}}\left(\left|Z_{1}\right|^{2}+\left|Z_{2}^{2}\right|\right) \sum_{\mathrm{PV}} \beta_{\mathrm{PV}}\left(1-\alpha_{\mathrm{PV}}\right)\left(2-\alpha_{\mathrm{PV}}\right)\left(\frac{m_{\mathrm{PV}}}{m_{\mathrm{PL}}}\right)^{2},
$$

where we have set the Kähler moduli to their self-dual value of $\left\langle t^{i}\right\rangle=1$ in reduced Planck-mass units and taken a dilaton-dominated scenario in which $F^{s} \bar{F}^{\bar{s}} K_{s \bar{s}}=F^{s} \bar{F}^{\bar{s}} K_{s} K_{\bar{s}}=3 m_{3 / 2}^{2}$ such that the diagonal entries of the lepton doublet scalar mass matrix are given by $m_{3 / 2}^{2}$ (see Appendix A below for a complete expression). Even in the case where $\alpha_{\mathrm{PV}} \neq 1,2$, and the summation is over PV fields whose masses are very near the string scale, it is still unlikely that the quadratically divergent contribution to FCNC in the lepton sector would be observable except in the case of very light scalar leptons or very large numbers of right-handed neutrino fields. In more complicated supersymmetry-breaking scenarios the exact nature of the FCNC bound is a more involved, modeldependent issue. Phenomenological models which introduce higher-order terms into the Kähler potential in the spirit of (108) [56, 63, 64, 65] should consider such loop-induced contributions to FCNC processes.

\section{Conclusion}

In this work we have considered those corrections to the scalar potential which are quadratically dependent on the cut-off scale. We have taken care to regulate theses divergences with a regulator sector that preserves manifest supersymmetry. We have argued that this step, overlooked in the past, is critical to a reliable discussion of the physical implications of these corrections.

Specifically, these quadratically divergent contributions to the effective scalar potential have two immediate impacts. The first is in the determination of the vacuum expectation values of various scalar fields in the low-energy four-dimensional theory. Of most importance are those fields whose auxiliary fields acquire supersymmetry breaking expectation values, as the vacuum values of these fields will generally dominate the vacuum expectation value of the potential itself; i.e. the vacuum energy of the cosmos. We have shown that the sign of the loop-induced contribution to this vacuum energy is not unambiguously positive and thus cannot be relied upon to remedy moduli stabilization mechanisms that produce substantial negative vacuum energy, such as the so-called "racetrack" method. In fact, the sign and magnitude of this contribution is model-dependent, 
but the casting of the problem in terms of a renormalization of the spacetime metric and Kähler potential - possible only when a manifestly supersymmetric regulating scheme is employed - makes this model-dependence easy to extract. We demonstrated this for the case of dilaton stabilization in the BGW model, for which the loop corrections were shown to affect the resulting minimum in a negligible way.

The second, and potentially more damaging, impact of these terms is on the generation of new contributions to the soft supersymmetry-breaking scalar mass matrices. In this case the constraints on the size of the off-diagonal entries of these matrices from rare flavor-changing neutral current (FCNC) processes makes these new contributions large enough to be worrisome if a summation over chiral fields can be sufficient to overcome the loop suppression factor. Of course, string theory (or any theory of high-scale physics) must still meet the challenge of explaining the smallness of scalar-mediated FCNC effects at the tree-level, but as string theory is as yet unable to address the Kähler potential for matter fields beyond the leading order we must ask instead for the ways in which supergravity can spoil such a safe arrangement once it is engineered.

When viewing this particular manifestation of the supersymmetric flavor problem from a groundup, phenomenological point of view it has been common to turn away from models in which supergravity plays a relevant role in low-energy physics so as to mitigate these new corrections. For example, gauge meditation of supersymmetry breaking is often promoted on this basis, and it purports to address the problem from two fronts: (1) by making the tree-level soft masses of the scalars proportional to gauge charges and (2) by allowing for a drastic reduction in the size of the gravitino mass - and thus in the size of supergravity induced soft-mass corrections of the form of (11).

For those who take a top-down, string theory motivated point of view the situation is less dire. String-based models are seldom "generic" and rarely give rise to the most general possible supergravity effective theory. The possible Kähler manifolds for the light scalars are typically quite limited form. In particular, isometries such as $S L(2, Z)$ symmetries amongst the various moduli (a specific form of the general Kähler transformation symmetry of supergravity Lagrangians) restrict the form of the tensors appearing in the expressions for off-diagonal scalar masses. Dangerously large terms which mix generations are likely to result only when particular higher-order terms are present or when particular moduli are involved in supersymmetry breaking. Specifically, terms of three or more fields with mixed holomorphicity tend to be the only ones of concern. Yet large coefficients are unlikely due to gauge-invariance constraints, except for a potential quartic term in the Kähler potential. We point out that even such quartic terms tend to count only those scalar fields within a particular sector of the string Hilbert space as opposed to all scalar fields in the 
theory - a difference that can reduce the possible size of these corrections substantially and which can only be appreciated by study of such terms within the context of realistic string constructions.

It also crucially important to consider the impact of the theory which serves as the ultraviolet (UV) completion for the supergravity effective theory. As we demonstrate, employing a supersymmetric regularization scheme introduces factors which must be treated as parameters in the effective theory. These represent the uncertainties in the threshold scale at which the UV physics (here represented by the Pauli-Villars sector) begins to operate to regularize divergences. The overall size of any supergravity-induced correction to scalar mass matrices ultimately depends on these factors in a model-dependent manner.

Even more crucial are the transformation properties of the regularization sector under the set of gauged $U(1)_{R}$ symmetries that are realized by Kähler transformations. The "charges" of the regulating sector fields under these transformations are determined by the dependence of the kinetic terms on the Kähler potential - parameters we denoted by $\alpha$ in (37). Different assumptions about this dependence act like different regularization schemes. Some choices can make dangerous offdiagonal scalar masses vanish identically. Such a dependence is missed by treatments that use supersymmetry-breaking (and modular symmetry-breaking) straight cut-off parameters and ignore the field dependence of these cut-offs.

Can we dispense with this intrusion of Planck-scale physics upon the quantities that interest us as low-energy observers? It is important to recognize that the UV-dependence is inherently necessary for the consistency of the theory. Consider, for example, the case of anomalous $U(1)_{X}$ symmetries in flat (rigid) supersymmetry. In the presence of such an anomalous $U(1)_{X}$ with nonvanishing $\operatorname{Tr} T_{X}$ there arises a quadratically divergent contribution at one loop proportional to $\operatorname{Tr} T_{X} \Lambda^{2}$. This contribution can not be cancelled by $U(1)_{X}$-invariant PV mass terms; that is, the mass terms in (13) must involve fields whose $U(1)_{X}$ charges do not cancel in order to generate a contribution to the quadratic divergence. Since the kinetic terms for these fields are given by $\exp \left(q_{X} V_{X}\right)|\Phi|^{2}$, where $V_{X}$ is the $U(1)_{X}$ real vector field, their masses in (14) are necessarily of the form $M=\exp \left(-q_{X} V_{X}\right) \mu$ and are field-dependent. Anomaly cancellation thus imposes constraints on the charges $q_{X}$.

The situation with Kähler transformations is completely analogous, as these transformations also involve a form of $U(1)$ transformation that is anomalous at the quantum level. In the presence of this Kähler anomaly there is a term

$$
\mathcal{L}_{\text {one-loop }} \ni \alpha K_{i \bar{m}} \mathcal{D}_{\mu} z^{i} \mathcal{D}^{\mu} \bar{z}^{\bar{m}} \Lambda^{2}
$$


which can only be cancelled if there is at least some sector of the PV fields for which $M_{\mathrm{PV}}^{2} \propto e^{\alpha K}$. Considering the mass term of (14) we see that the PV masses can indeed be made invariant under Kähler transformations if $\alpha_{A}+\alpha_{B}=1$ for pairs of fields $\Phi^{A}, \Pi^{B}$ appearing in the superpotential terms in (13). Indeed, this choice gives rise to precisely the relationship in (15) ${ }^{14}$ This cannot be true of all fields in the PV sector, however, since if so the contribution of the PV sector to Tr $H^{2}$ is always proportional to $\frac{r}{2}+K_{i \bar{m}} \mathcal{D}_{\mu} z^{i} \mathcal{D}^{\mu} \bar{z}^{\bar{m}}-2 V$ which can be removed by a Weyl redefinition. That is, this expression vanishes identically on-shell when the graviton equations of motion are employed. Therefore more general choices of the various $\alpha$ values are needed to cancel these terms for the full regularization of all divergences (which imposes certain constraints as derived in Section 2.2) and to insure that the Kähler chiral anomaly has a trace anomaly superpartner, as in the $U(1)_{X}$ case described above.

In both of these examples the elements of the one-loop Lagrangian that cannot be cancelled without field-dependent masses of the form $m \sim e^{p V}$, where $p$ is a generalized "weight" and $V$ is a real superfield, are elements that arise via anomalies. Therefore we have no reason to expect that the low-energy physics should be independent of these weights $p$ : it is, after all, precisely this property of anomalies that make them so useful in connecting UV and IR physics. The results presented in this work are thus far more than an academic exercise about finding the appropriate coefficients for a complicated loop calculation, but are at the heart of how supergravity effective Lagrangians connect low-scale physics and high-energy assumptions in phenomenologically meaningful ways.

\section{Acknowledgements}

We would like to thank G. Kane, J. Giedt and P. Ko for encouragement and helpful discussions in early stages of this work. MKG was supported in part by the Department of Energy under grant DE-AC02-05CH11231 and in part by the National Science Foundation under grant PHY-0098840. BDN was supported by the Department of Energy under grant EY-76-02-3071.

\section{Appendix A - General Scalar Mass Formulae}

For the sake of completeness, in this appendix we present the complete scalar mass expression obtained from inserting the appropriate matrix elements into expression (92) and inserting the result into the corrected weighted sum. To keep the expression tractable we work only to first order in the

\footnotetext{
${ }^{14}$ This is the configuration dubbed "PV Scenario A" in the phenomenological treatment of 35].
} 
loop expansion parameter and assume vanishing tree-level vacuum energy (i.e. $K_{n \bar{m}} F^{n} \bar{F}^{\bar{m}}=3 m_{3 / 2}^{2}$ ). Then the scalar squared mass is given by

$$
\begin{aligned}
\left(m^{2}\right)_{b}^{a}= & m_{3 / 2}^{2}\left[\delta_{b}^{a}-\frac{3}{32 \pi^{2}}\left(\delta_{b}^{a} \sum_{p} \zeta_{P} \eta_{p} \tilde{\alpha}_{p}^{2} \ln \left(\beta_{p}^{2}\right) \beta_{p}^{2} \Lambda_{p}^{2}-K^{a \bar{c}} R_{b \bar{c}} \sum_{\alpha} \eta_{\alpha} \tilde{\alpha}_{\alpha} \ln \left(\beta_{\alpha}^{2}\right) \beta_{\alpha}^{2} \Lambda_{\alpha}^{2}\right)\right] \\
& +R_{n \bar{m} b}^{a} F^{n} \bar{F}^{\bar{m}}\left(1+\frac{1}{2} \delta K-\frac{3}{32 \pi^{2}} \sum_{p} \zeta_{P} \eta_{p} \tilde{\alpha}_{p} \ln \left(\beta_{p}^{2}\right) \beta_{p}^{2} \Lambda_{p}^{2}\right) \\
& -\frac{1}{32 \pi^{2}} \sum_{p} \zeta_{P} \eta_{p}\left[\left(\bar{v}_{\bar{m}}^{p} \bar{F}^{\bar{m}}-m_{3 / 2}\right) v_{p}^{\bar{l}} F^{n} R_{n \bar{l} b}^{a}+\text { h.c. }\right] \ln \left(\beta_{p}^{2}\right) \beta_{p}^{2} \Lambda_{p}^{2} \\
& +\frac{1}{32 \pi^{2}} F^{n} \bar{F}^{\bar{m}}\left(K^{a \bar{c}} R_{b \bar{c}} R_{n \bar{m} b}^{d}+R_{n \bar{l}} R_{\bar{m} b}^{a}{ }^{\bar{l}}+R_{l \bar{m}} R_{n b}^{a}{ }_{b}\right) \sum_{\alpha} \eta_{\alpha} \ln \left(\beta_{\alpha}^{2}\right) \beta_{\alpha}^{2} \Lambda_{\alpha}^{2} \\
& +\frac{1}{32 \pi^{2}}\left[\frac{F^{n} \bar{F}^{\bar{m}}}{4 x^{2}}\left(f_{n} \bar{f}_{\bar{l}} R_{\bar{m} b}^{a}{ }_{\bar{l}}+f_{l} \bar{f}_{\bar{m}} R_{n b}^{a}{ }_{n}{ }^{2}\right)-m_{1 / 2}^{2}\right] \sum_{\alpha} \eta_{\alpha}^{\varphi} \ln \left(\beta_{\alpha}^{\varphi}\right)^{2}\left(\beta_{\alpha}^{\varphi}\right)^{2} \Lambda_{\varphi}^{2} \\
& -\frac{1}{32 \pi^{2}} F^{n} \bar{F}^{\bar{m}} K^{a \bar{c}} \sum_{\alpha}\left(D_{\bar{m}} D_{b} R_{n \bar{c}}+\bar{v}_{\bar{m}}^{\alpha} D_{b} R_{n \bar{c}}+v_{n}^{\alpha} D_{\bar{m}} R_{b \bar{c}}+v_{n}^{\alpha} \bar{v}_{\bar{m}}^{\alpha} R_{b \bar{c}}\right) \eta_{\alpha} \ln \left(\beta_{\alpha}^{2}\right) \beta_{\alpha}^{2} \Lambda_{\alpha}^{2} \\
& -\frac{\delta_{b}^{a}}{16 \pi^{2}} F^{n} \bar{F}^{\bar{m}} R_{n \bar{m}} \sum_{\alpha} \eta_{\alpha} \tilde{\alpha}_{\alpha} \ln \left(\beta_{\alpha}^{2}\right) \beta_{\alpha}^{2} \Lambda_{\alpha}^{2}-\frac{1}{32 \pi^{2}} \sum_{p} \zeta_{P} \eta_{p} \alpha_{p}\left|v_{n}^{p} F^{n}\right|^{2} \ln \left(\beta_{p}^{2}\right) \beta_{p}^{2} \Lambda_{p}^{2}, \quad(114) \quad
\end{aligned}
$$

where

$$
\begin{aligned}
\tilde{\alpha}_{p} & =\left(\tilde{\alpha}_{\alpha}, \widehat{\tilde{\alpha}}_{\alpha}, \tilde{\alpha}_{\alpha}^{\phi}, \tilde{\alpha}_{b}^{\theta}\right)=\left(1-\alpha_{\alpha},-\hat{\alpha}_{\alpha}, 0, \alpha_{b}^{\theta}\right), \\
v_{i}^{\alpha} & =\tilde{\alpha}_{\alpha} K_{i}-\partial_{i} \mu-\Gamma_{k i}^{k}, \quad\left(v_{\alpha}^{\varphi}\right)_{i}=K_{i}-\frac{f_{i}}{2 x}-\partial_{i} \mu_{\varphi}, \\
v_{i}^{p} & =\tilde{\alpha}_{p} K_{i}-\partial_{i} \mu_{P}, \quad P_{p}=\widehat{\Phi}, \widehat{\Pi}, \theta_{b}, \quad v_{p}^{\bar{m}}=K^{\bar{m} i} v_{i}^{p} .
\end{aligned}
$$

If we take the specific case of Kähler potential given by (101)

$$
K=g(M, \bar{M})+\sum_{a} f_{a}(M, \bar{M})\left|\Phi^{a}\right|^{2}+\frac{1}{4} \sum_{a b} X_{a b} f_{a} f_{b}\left|\Phi^{a}\right|^{2}\left|\Phi^{b}\right|^{2}+O\left(|\Phi|^{3}\right),
$$

which was also the example considered in [10], we obtain for the diagonal scalar mass entries

$$
\begin{aligned}
m_{a}^{2}= & m_{3 / 2}^{2}\left[1-\frac{3}{32 \pi^{2}}\left(\sum_{p} \zeta_{P} \eta_{p} \tilde{\alpha}_{p}^{2} \ln \left(\beta_{p}^{2}\right) \beta_{p}^{2} \Lambda_{p}^{2}-\left(\sum_{i} q_{i}^{a}+\sum_{b} X_{a b}\right) \sum_{\alpha} \eta_{\alpha} \tilde{\alpha}_{\alpha} \ln \left(\beta_{\alpha}^{2}\right) \beta_{\alpha}^{2} \Lambda_{\alpha}^{2}\right)\right] \\
& -q_{i}^{a} g_{i \bar{\jmath}} F^{i} \bar{F}^{\bar{\jmath}}\left(1+\frac{1}{2} \delta K-\frac{3}{32 \pi^{2}} \sum_{p} \zeta_{P} \eta_{p} \tilde{\alpha}_{p} \ln \left(\beta_{p}^{2}\right) \beta_{p}^{2} \Lambda_{p}^{2}\right) \\
& +q_{i}^{a} \frac{1}{32 \pi^{2}} \sum_{p} \zeta_{P} \eta_{p}\left[\left(\bar{v}_{\bar{m}}^{p} \bar{F}^{\bar{m}}-m_{3 / 2}\right) v_{i}^{p} F^{i}+\text { h.c. }\right] \ln \left(\beta_{p}^{2}\right) \beta_{p}^{2} \Lambda_{p}^{2}
\end{aligned}
$$




$$
\begin{aligned}
&-\frac{1}{32 \pi^{2}} g_{i \bar{\jmath}} F^{i} \bar{F}^{\bar{\jmath}}\left[2 \tilde{\alpha}\left(2+\sum_{b} q_{i}^{b}\right)-\sum_{b} q_{i}^{b} X_{a b}\right. \\
&\left.-2 q_{i}^{a}\left(2+\sum_{b} X_{a b}+\sum_{k} q_{k}^{a}+\sum_{b} q_{i}^{b}\right)\right] \sum_{\alpha} \eta_{\alpha} \ln \left(\beta_{\alpha}^{2}\right) \beta_{\alpha}^{2} \Lambda_{\alpha}^{2} \\
&-\frac{1}{32 \pi^{2}}\left[m_{1 / 2}^{2} \sum_{\alpha} \eta_{\alpha}^{\varphi} \ln \left(\beta_{\alpha}^{\varphi}\right)^{2}\left(\beta_{\alpha}^{\varphi}\right)^{2} \Lambda_{\varphi}^{2}+\sum_{i} q_{i}^{a} \sum_{\alpha}\left|v_{n}^{\alpha} F^{n}\right|^{2} \eta_{\alpha} \ln \left(\beta_{\alpha}^{2}\right) \beta_{\alpha}^{2} \mid \Lambda_{\alpha}^{2}\right] \\
&-\frac{1}{32 \pi^{2}}\left[\sum_{b}\left(q_{i}^{a}+q_{i}^{b}\right) X_{a b}+q_{i}^{b} \sum_{k} q_{k}^{a}\right]\left(g_{i} F^{i} \bar{F}^{\bar{m}} \sum_{\alpha} \bar{v}_{\bar{m}}^{\alpha}+\text { h.c. }\right) \eta_{\alpha} \ln \left(\beta_{\alpha}^{2}\right) \beta_{\alpha}^{2} \mid \Lambda_{\alpha}^{2} \\
&-\frac{\delta_{b}^{a}}{16 \pi^{2}} F^{s} \bar{F}^{\bar{s}} R_{s \bar{s}} \sum_{\alpha} \eta_{\alpha} \tilde{\alpha}_{\alpha} \ln \left(\beta_{\alpha}^{2}\right) \beta_{\alpha}^{2} \Lambda_{\alpha}^{2}-\frac{1}{32 \pi^{2}} \sum_{p} \zeta_{P} \eta_{p} \alpha_{p}\left|v_{n}^{p} F^{n}\right|^{2} \ln \left(\beta_{p}^{2}\right) \beta_{p}^{2} \Lambda_{p}^{2},
\end{aligned}
$$

where $n, m=s, i, j, k$ and $i, j, k$ label T-moduli.

\section{Appendix B - Errata}

In this appendix we take a moment to correct a handful of errata in some of the papers referred to in this work:

Ref. [26] In the first line of Eq. (7) the term $-\frac{r}{2}$ should be replaced by $+\frac{7 r}{2}$. This entails a correction to Eqs. (15), (16) and (20) of that paper; the correct relations are given in [32, 33. In Eq. (17) there should be a replacement $\widehat{V} \rightarrow V$ in the first term on the right-hand side. In this same equation, the following term

$$
\frac{1}{2} H_{b}^{2} \ni \frac{4}{x x_{b}}\left|\delta^{b} \theta^{b}\right|^{2} K_{b \bar{b}} \mathcal{D}_{a} D_{b}\left(T^{a} z\right)^{b}=-\frac{1}{x} \delta k_{b} \Gamma_{b k}^{b}\left(T^{a} z\right)^{k} \mathcal{D}_{a}=-2 \alpha_{b}^{\theta} \mathcal{D} \delta k_{b} .
$$

is missing and should be inserted. This term can be spotted in the 4th term on the right-hand side of Eq. (C.36) in [19]; it corresponds to the last term in Eq. (C.37) of the same reference.

Ref. [18] In the expression for $L_{0}$ in Eq. (13), the coefficient of $\frac{r}{2}$ in the first term should be $\left(N_{G}+7\right)$ instead of $\left(N_{G}-1\right)$ and the second term should have $2 K_{i \bar{m}}\left(\mathcal{D}_{\mu} z^{i} \mathcal{D}^{\mu} \bar{z}^{\bar{m}}+5 F^{i} \bar{F}^{\bar{m}}\right)$ replaced by $2 K_{i \bar{m}}\left(2 \mathcal{D}_{\mu} z^{i} \mathcal{D}^{\mu} \bar{z}^{\bar{m}}-5 F^{i} \bar{F}^{\bar{m}}\right)$.

\section{References}

[1] J. R. Ellis and D. V. Nanopoulos, Phys. Lett. B 110, 44 (1982). 
[2] J. F. Donoghue, H. P. Nilles and D. Wyler, Phys. Lett. B 128, 55 (1983).

[3] J. Louis and Y. Nir, Nucl. Phys. B 447, 18 (1995).

[4] P. H. Chankowski, O. Lebedev and S. Pokorski, Nucl. Phys. B 717, 190 (2005).

[5] O. Lebedev, "A stringy solution to the FCNC problem," [hep-ph/0506052].

[6] R. Barbieri, S. Ferrara and C. A. Savoy, Phys. Lett. B 119, 343 (1982).

[7] A. H. Chamseddine, R. Arnowitt and P. Nath, Phys. Rev. Lett. 49, 970 (1982).

[8] P. Binetruy, G. L. Kane, J. D. Lykken and B. D. Nelson, "Twenty-five questions for string theorists," (2005) [arXiv:hep-th/0509157].

[9] K. Choi, J. E. Kim and H. P. Nilles, Phys. Rev. Lett. 73, 1758 (1994).

[10] K. Choi, J. S. Lee and C. Munoz, Phys. Rev. Lett. 80, 3686 (1998).

[11] S. R. Coleman and E. Weinberg, Phys. Rev. D 7, 1888 (1973).

[12] M. Srednicki and S. Theisen, Phys. Rev. Lett. 54, 278 (1985).

[13] J. D. Breit, B. A. Ovrut and G. Segre, Phys. Lett. B 162, 303 (1985).

[14] P. Binetruy and M. K. Gaillard, Phys. Lett. B 168, 347 (1986).

[15] C. Chiou-Lahanas, A. Kapella-Economu, A. B. Lahanas and X. N. Maintas, Phys. Rev. D 42, 469 (1990).

[16] C. Chiou-Lahanas, A. Kapella-Economou, A. B. Lahanas and X. N. Maintas, Phys. Rev. D 45, 534 (1992).

[17] M. K. Gaillard and V. Jain, Phys. Rev. D 49, 1951 (1994).

[18] M. K. Gaillard, V. Jain and K. Saririan, Phys. Lett. B 387, 520 (1996).

[19] M. K. Gaillard, V. Jain and K. Saririan, Phys. Rev. D 55, 883 (1997).

[20] P. Binetruy, S. Dawson, M. K. Gaillard and I. Hinchliffe, Phys. Rev. D 37, 2633 (1988).

[21] J. W. Burton, M. K. Gaillard and V. Jain, Phys. Rev. D 41, 3118 (1990). 
[22] J. Giedt, Annals Phys. 297, 67 (2002).

[23] P. Binetruy and M. K. Gaillard, Phys. Lett. B 220, 68 (1989).

[24] P. Binetruy and M. K. Gaillard, Nucl. Phys. B 358, 121 (1991).

[25] M. K. Gaillard and T. R. Taylor, Nucl. Phys. B 381, 577 (1992).

[26] M. K. Gaillard, Phys. Lett. B 342, 125 (1995).

[27] A. Birkedal, M. K. Gaillard, C. Park and M. Ransdorp, paper in preparation.

[28] P. Binetruy and M. K. Gaillard, Nucl. Phys. B 312, 341 (1989).

[29] I. Antoniadis, K. S. Narain and T. R. Taylor, Phys. Lett. B 267, 37 (1991).

[30] L. J. Dixon, V. Kaplunovsky and J. Louis, Nucl. Phys. B 355, 649 (1991).

[31] K. Choi, H. B. Kim and C. Munoz, Phys. Rev. D 57, 7521 (1998).

[32] M. K. Gaillard, Phys. Rev. D 58, 105027 (1998).

[33] M. K. Gaillard, Phys. Rev. D 61, 084028 (2000).

[34] M. K. Gaillard and B. D. Nelson, Nucl. Phys. B 588, 197 (2000).

[35] P. Binetruy, M. K. Gaillard and B. D. Nelson, Nucl. Phys. B 604, 32 (2001).

[36] P. Binetruy, M. K. Gaillard and Y. Y. Wu, Nucl. Phys. B 493, 27 (1997).

[37] P. Binetruy, M. K. Gaillard and Y. Y. Wu, Phys. Lett. B 412, 288 (1997).

[38] P. Binetruy, M. K. Gaillard and Y. Y. Wu, Nucl. Phys. B 481, 109 (1996).

[39] J. A. Casas, Phys. Lett. B 384, 103 (1996).

[40] S. H. Shenker, "The Strength Of Nonperturbative Effects In String Theory," RU-90-47 Presented at the Cargese Workshop on Random Surfaces, Quantum Gravity and Strings, Cargese, France, May 28 - Jun 1, 1990.

[41] E. Silverstein, Phys. Lett. B 396, 91 (1997).

[42] T. Banks and M. Dine, Phys. Rev. D 50, 7454 (1994). 
[43] J. Giedt and B. D. Nelson, JHEP 0405, 069 (2004).

[44] M. K. Gaillard and B. D. Nelson, Nucl. Phys. B 571, 3 (2000).

[45] A. Birkedal-Hansen and B. D. Nelson, Phys. Rev. D 64, 015008 (2001).

[46] A. Birkedal-Hansen and B. D. Nelson, Phys. Rev. D 67, 095006 (2003).

[47] G. L. Kane, J. D. Lykken, B. D. Nelson and L. T. Wang, Phys. Lett. B 551, 146 (2003).

[48] T. Barreiro, B. de Carlos, J. A. Casas and J. M. Moreno, Phys. Lett. B 445, 82 (1998).

[49] M. K. Gaillard, J. Giedt and A. L. Mints, Nucl. Phys. B 700, 205 (2004) [Erratum-ibid. B 713, 607 (2005)].

[50] E. Witten, Nucl. Phys. B 471 (1996) 135.

[51] A. Font, L. E. Ibanez, F. Quevedo and A. Sierra, Nucl. Phys. B 331, 421 (1990).

[52] L. E. Ibanez and D. Lust, Nucl. Phys. B 382, 305 (1992).

[53] G. L. Kane, J. D. Lykken, S. Mrenna, B. D. Nelson, L. T. Wang and T. T. Wang, Phys. Rev. D 67, 045008 (2003).

[54] G. L. Kane, T. T. Wang, B. D. Nelson and L. T. Wang, Phys. Rev. D 71, 035006 (2005).

[55] G. F. Giudice and A. Masiero, Phys. Lett. B 206, 480 (1988).

[56] S. Abel, A. Dedes and K. Tamvakis, Phys. Rev. D 71, 033003 (2005).

[57] J. Giedt, G. L. Kane, P. Langacker and B. D. Nelson, Phys. Rev. D 71, 115013 (2005).

[58] P. Langacker and B. D. Nelson, Phys. Rev. D 72, 053013 (2005).

[59] M. L. Brooks et al. [MEGA Collaboration], Phys. Rev. Lett. 83, 1521 (1999).

[60] S. Lavignac, "Flavour and CP violation in the lepton sector and new physics," eConf C030603, VEN04 (2003) [arXiv:hep-ph/0312309].

[61] F. Gabbiani, E. Gabrielli, A. Masiero and L. Silvestrini, Nucl. Phys. B 477, 321 (1996). 
[62] J. L. Feng, "Theoretical motivations for lepton flavor violation," (2001) [arXiv:hep$\mathrm{ph} / 0101122]$.

[63] J. A. Casas, J. R. Espinosa and I. Navarro, Phys. Rev. Lett. 89, 161801 (2002).

[64] J. A. Casas, J. R. Espinosa and I. Navarro, JHEP 0309, 048 (2003).

[65] J. March-Russell and S. M. West, Phys. Lett. B 593, 181 (2004). 